Originalveröffentlichung in: Dietrich Boschung, Christiane Vorster (Hg.), Leibhafte Kunst. Statuen und kulturelle Identität (Morphomata 24), Paderborn 2015, S. 13-53

TONIO HÖLSCHER (HEIDELBERG)

\title{
DIE ENTSTEHUNG DER GRIECHISCHEN POLISGEMEINSCHAFT IM BILD. LEBENDE, VORFAHREN, GÖTTER
}

The creation of large-scale statues in stone, bronze, and wood in early Greece indicates an historical process with enormous consequences. Learning from the great cultures of Egypt and the Near East and honing their skills on indigenous marble, Greek sculptors developed forms of monumental sculpture equal to those of the neighbouring cultures.

In early Greek art large-scale statues were intended for public spaces, where they visualized three different subjects: gods as cult images in temples, typified humans as votive dedications in sanctuaries, and the dead as commemorative images at tombs. Within the context of early Greek life these monumental images served a fundamental purpose. The early Greek polis was an ideal community to which living people, deceased ancestors, and the gods equally belonged. The interaction of the living with those ideal community members took place in the public spaces of early cities; these early political constructs were, in fact, constituted by public spaces. Male citizens convened in the assembly on the agora, while the entire citizenry developed their idealized life structures on the occasion of festivals for the gods in their sanctuaries and of rituals for the dead in the necropolises. There the statues had the task to establish the presence of those community »members" that were not actually present: gods in temples, model representatives of the community in sanctuaries, the dead at their tombs. To the Greeks, the statues embodied these figures in a very immediate and concrete way: they enabled the living to interact with the other members of the ideal community in rituals and other concrete forms of communication.

In the communities of the early polis with their low-level hierarchies these forms of »face-to-facer interaction and »immediate action" had the 
result that symbolic signs of social status such as dress or insignia were less important than the immediate effect of bodily appearance. In this sense, a winning and convincing appearance became a key characteristic both in social reality and the visual arts: physical strength, agility, and the beauty of the naked body for men; erotic charm in both dress and conduct for women. The statues were effective components in the visual self-definition of the emerging Greek polis.

\section{BILDWERKE, ÖFFENTLICHKEIT UND POLIS}

Ein Reisender, der eine antike Stadt besuchte, fand sich, neben öffentlichen Gebäuden und privaten Häusern, einer ungemein großen Zahl von Bildwerken gegenüber: Schon vor der Stadtmauer waren die $\mathrm{Zu}-$ gangsstraßen von Nekropolen mit den Grabstatuen und Grabreliefs der reichen Familien gesäumt; in den städtischen Heiligtümern standen die Kultbilder der Götter im Inneren der Tempel und die statuarischen Weihgeschenke in den umgebenden Bezirken; auf der Agora erhoben sich die politischen Denkmäler. Hinzu kamen später Bildwerke in öffentlichen Bauten wie Rathäusern, Theatern, Gymnasien, in römischer Zeit vor allem auch in Thermenanlagen. Jeder dieser städtischen Lebensräume hatte seine zugehörigen Bildwerke.

Wenn man der Einzigartigkeit dieser griechischen - und in ihrer Folge der römischen - Bildkultur nachgeht, dann stellt sich die grundsätzliche Frage: Warum haben die Griechen überhaupt diese überwältigende Menge von Bildwerken gebraucht? Es liegt nahe, mit dieser Frage insbesondere die griechische Frühzeit zu betrachten, in der die Praktiken dieser Bildkultur entstanden sind.

Die Griechen hatten seit dem frühen 1. Jahrtausend v. Chr. eine reiche, differenzierte Bildkunst in kleinem Format ausgebildet: Tongefäße mit vielfältigen Bildszenen, Statuetten aus Bronze, Terrakotta, sogar Elfenbein, die in den zentralen sozialen Situationen eingesetzt wurden: bei Hochzeit und Symposion, dazu im Kult für die Götter und die Toten. Diese Kunst konnte man weiter entwickeln, und man hat das auch getan, bis zum Ende der Antike. Im frühen bis mittleren 7. Jahrhundert v. Chr. begann man aber plötzlich, Bildwerke in großem Format, oft knapp unter, bald aber auch über Lebensgröße herzustellen. Erhalten sind Standbilder aus Marmor und Kalkstein, ferner Reste großer Figuren aus getriebenem Bronzeblech; daneben muss man von einer reichen Kunst in Holz ausgehen, die sich kaum erhalten hat, die aber zu einer großen 
Kunstfertigkeit gesteigert wurde. Was leisteten solche Bildwerke gegenüber denen in kleinem Format?

Der entscheidende Schritt besteht darin, dass das Bild dem betrachtenden Menschen gleichrangig und ebenbürtig, gewissermaßen als Partner, gegenüber steht. Man kann das Standbild nicht nach Belieben hin und her und aus dem Weg räumen, das Bild steht von sich aus im Raum, es wendet sich an potentielle Betrachter, es fordert deren Aufmerksamkeit. Wenn schon bald Epigramme ein Bildwerk begleiten, dann sprechen sie im Namen der dargestellten Person ein Publikum an: "Schau mich an, wie schön ich bin!« Das heißt: Bildwerke von großem Format bedeuten eine neue, starke Form von Publizität. ${ }^{2}$

In der Tat hat die griechische Skulptur in großem Format sich ausschließlich in den öffentlichen Räumen entwickelt, wo die Standbilder starke Sichtbarkeit erreichten: Schon die frühesten großen Bildwerke sind Götterbilder in Tempeln, Weihgeschenke in Heiligtümern und Figuren der Verstorbenen über den Gräbern; später kam die Agora als Raum von Standbildern hinzu. Die großen Heiligtümer waren Orte der Zusammenkunft der städtischen Gemeinschaft, die Agora war der Platz der politischen Versammlungen, und auch die Nekropolen waren keine abgeschiedenen Friedhöfe, sondern säumten die Zugangsstraßen zur Stadt vor den Toren, wo sie die Aufmerksamkeit von ankommenden und aufbrechenden Reisenden fanden (Abb. 1).

Damit ist der historische Rahmen bezeichnet, in dem der Schritt zur großformatigen Skulptur zu verstehen ist. Es ist die Entstehung der charakteristischen Staatsform des frühen Griechenland: der Polis, des Stadtstaates. Die erste entscheidende Phase dieser Entwicklung gehört ins 8. und 7. Jahrhundert v. Chr. Damals formierten sich aus dörflichen Gemeinden, in denen mächtige Familien mit ihren Oberhäuptern weitgehend autonom nebeneinander lebten, größere Gemeinwesen, die ihre Angelegenheiten und Interessen gemeinschaftlich zu regeln unternahmen. Die sstädtischen ' Zentren, in denen sie sich zusammenschlossen, erhielten nun Strukturen der Gemeinschaftlichkeit, und in diesem Sinn

\footnotetext{
$1 \mathrm{Zu}$ den Anfängen der griechischen Großplastik s. die erste umfassende Untersuchung von Homann-Wedeking 1950. Neuere Arbeiten: Ridgway 1977, 17-42; Fuchs - Floren 1987; Martini 1990, 101-131; Stewart 1990, 103-110; Rolley 1994, 116-154; Vorster 2002; Brisart 2011, 273-314. Statuen aus Holz: Herrmann 1975. Bilder aus getriebenem Bronzeblech: Borell - Rittig 1998. 2 Basen von Bildwerken mit Epigrammen, vor allem von Verstorbenen am Grab: Kissas 2000.
} 


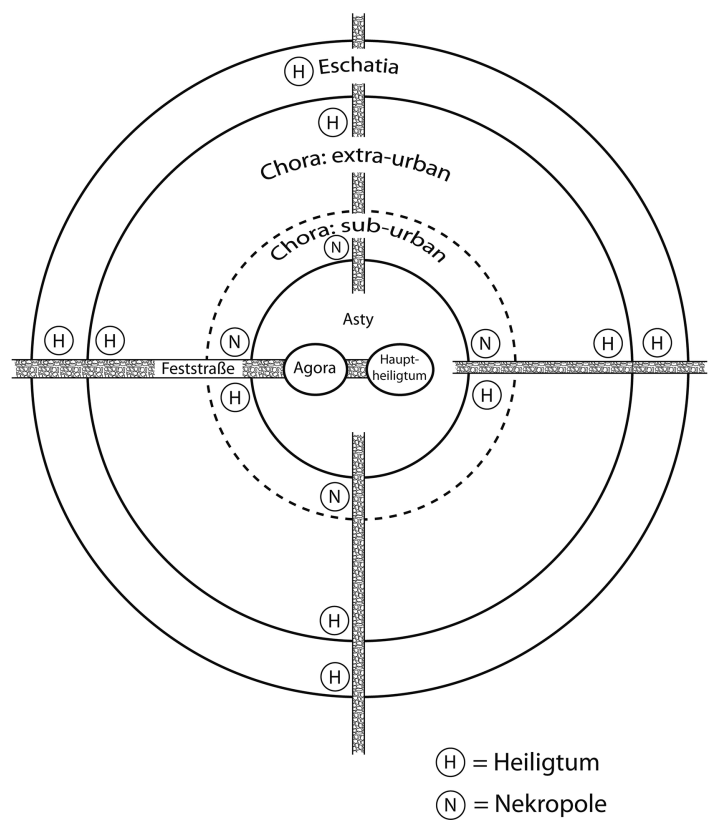

1 Räume der griechischen Polis

der `Öffentlichkeit`, die etwa am Beispiel von Athen oder Argos deutlich werden. Zum einen entstanden zentrale Heiligtümer, wo die `Bürger sich zum gemeinsamen Kult und Fest einfanden; zum anderen wurde ein zentraler politischer Versammlungsplatz eingerichtet, die Agora, wo die politische Versammlung der männlichen Bürger zusammenkam. Darüber hinaus wurden nun die Gräber, die bisher im Siedlungsgebiet nahe bei den Häusern gelegen hatten, in eigene Bezirke außerhalb des Wohngebietes verlegt. Damit erhielt zunächst die städtische Gemeinschaft einen gemeinsamen Siedlungsraum. Doch auch die Grabstätten wurden nicht in irgendein wildes 'Draußen ausgelagert, sondern vor allem entlang den großen Zugangs- und Ausfahrtstraßen der Stadt angelegt, wo sie ihrerseits einen kohärenten Raum von öffentlicher Sichtbarkeit erhielten. Jenseits dieser periurbanen Zone waren die Städte konzentrisch umgeben von der chora, dem Ackerland, und diese wiederum von der Grenzzone der freien Natur, Wälder und Berge, der eschatiá, wo wilde Tiere die menschliche Ordnung bedrohten. ${ }^{3}$

$3 \mathrm{Zu}$ dieser Grundstruktur der griechischen Polis s. Hölscher 1998. 
In dem Konzept der `Stadt` waren drei öffentliche Räume eingerichtet, in denen die Gemeinschaft der Polis mit den entscheidenden Mächten der Welt in Verbindung treten konnte: mit den Göttern durch den Kult in den Heiligtümern, mit den lebenden Mitbürgern in der politischen Versammlung auf der Agora und mit den Toten durch die Verehrung in den Nekropolen. Menschen, Götter und Tote bildeten eine konzeptuelle Gemeinschaft.

Diese Strukturierung der gemeinschaftlichen Räume im 8. bis 7. Jahrhundert v. Chr. war der entscheidende erste Schritt in der Neuformierung der griechischen Gesellschaft und Kultur nach dem Ende der mykenischen Palastzeit. Die räumlichen Anlagen bezeugen religiöse Rituale und soziale Praktiken politischer Proto-Gemeinschaften, die über die Struktur der Familien und ihrer Gefolgsleute hinausgingen. Ein zweiter Schritt folgte seit dem Anfang des 6. Jahrhunderts v. Chr. mit der Monumentalisierung der öffentlichen Räume. Vor allem die großen städtischen Heiligtümer wurden jetzt mit monumentalen Tempeln ausgestattet, die große gemeinsame Anstrengungen voraussetzen und damit eine politische Verdichtung der Bürgerschaften erkennen lassen.

Eben diese Räume waren es aber, in denen bald die ersten Bildwerke in großem Format errichtet wurden. Das ist zwar allgemein bekannt, aber die Konsequenzen daraus sind längst nicht entschieden genug gezogen worden. Entscheidend ist, dass wir uns grundsätzlich frei machen von der modernen Vorstellung des Museums als Ort einer ausschließlichen Betrachtung von $>$ Kunst $\triangleleft$. Antike Bildwerke gehörten in die Kontexte der Lebenswelt und waren Elemente der sozialen Praxis. Ihre Funktion bestand darin, Götter und Heroen der Vergangenheit ebenso wie frühere und gegenwärtige Mitglieder der Gemeinschaft in den Räumen des Lebens präsent zu machen, damit die Lebenden mit ihnen umgehen konnten. Es gab keine Museen, sondern Lebensräume, keine `Kunstwerke‘, sondern bildliche Lebewesen, und keine Betrachter, sondern Menschen, die mit den Bild-Wesen lebten. Götter und Heroen, Vorfahren und Lebende, im Bild wie in corpore vivo, bildeten die konzeptuelle Gemeinschaft der Polis. Es liegt darum nahe, den Schritt zur Bildkunst in großem Format im Rahmen der Formierung der Polisgesellschaften zu sehen. ${ }^{4}$

Dabei bezeugen die beiden Phasen in der Gestaltung öffentlicher Räume zwei Stufen in der Entwicklung der Polis. Im 8. und 7. Jahr-

4 Versuch einer Darstellung der griechischen Bildkunst im Rahmen sozialer Praxis: Hölscher 2007. 
hundert v. Chr. waren in den protopolitischen Gemeinschaften noch die Oberhäupter weniger führender Familien vorherrschend, die ihre Macht oft relativ unabhängig von den Interessen der Gemeinschaft ausbauten. Dem gegenüber gewannen seit Beginn des 6. Jahrhunderts v. Chr. breitere Oberschichten an Einfluss, die aristokratische Ansprüche und Lebensformen ausbildeten und allgemein eine dichtere >Bürgerlichkeit< bewirkten. In der Bildkultur hat das zur Folge, dass im 7. Jahrhundert v. Chr. zunächst nur vereinzelte Standbilder großen Formats errichtet wurden, während sich im 6. Jahrhundert v. Chr. in verschiedenen Städten eine breitere Praxis der Statuensetzung entwickelte.

Damit wird die Frage nach der Entstehung der griechischen Großplastik weit weg von den Wegen verlagert, auf denen die Forschung dies Phänomen zumeist zu verstehen suchte. Eine dieser Erklärungen betrifft die künstlerische Form: Sie weist auf Einfluss aus Ägypten oder dem Vorderen Orient hin. In der Tat haben die Griechen unbestreitbar von den dortigen Hochkulturen die Skulptur in großem Format und auch die wichtigsten Darstellungstypen für männliche und weibliche Figuren übernommen. Die Forschung hat das längst gesehen, und sie hat zugleich herausgearbeitet, wie die Vorbilder in Griechenland umgeformt wurden: Gegenüber ägyptischen Standbildern, die von einem Rückenpfeiler oder einer imaginären Rückenachse gehalten werden und ihr ausgreifendes Bein unorganisch nach vorne strecken, verteilen griechische Standbilder das Gewicht gleichmäßig auf beide Beine und stehen dadurch raus eigener Kraft $\measuredangle$. Die Forschung hat diese Unterschiede z. T. stark betont und darin sicher zu Recht eine typisch griechische Auffassung des Körpers gesehen, die dann zu der einzigartigen Entwicklung der griechischen Körper-Kunst geführt hat. ${ }^{5}$

Aus diesen und anderen Beobachtungen wird heute zunehmend die Folgerung gezogen, dass die Übernahme kultureller Elemente aus dem Orient nur äußerliche Anstöße seien, dass die eigentliche Leistung doch in der griechischen Neuformung bestehe, und dass darin ein wesentlicher Zug der kulturellen Identität des antiken Griechenland zum Ausdruck komme. Das ist aber ein eurozentrisches Konzept, das die Phänomene aus einer heutigen Perspektive beurteilt. Zum einen kann die Idee und die technische Bewältigung des großen Formats als solche, die die Griechen von den Nachbarkulturen übernahmen, mit guten Gründen für die größere kulturelle Leistung angesehen werden als die Umformungen,

5 Krahmer 1931; Himmelmann 1964, 18-21. 
die die Griechen zunächst daran vornahmen. Wenn in Griechenland führende Aristokraten und ihre Bildhauer begannen, Standbilder in großem Format wie in Ägypten und im Vorderen Orient zu errichten, so bezeugten sie damit sicher in erster Linie ihre Zugehörigkeit zu einer gemeinsamen Kultur, einer ostmediterranen koine, zu der Griechenland jetzt Zugang suchte, ${ }^{6} \mathrm{mehr}$ als eine eigene griechische >Identität‘.

Die Bildwerke wurden jedoch zunächst nicht als allgemeiner Ausdruck kultureller Zugehörigkeit, sondern für konkrete Aufgaben eingesetzt, und diese Aufgaben waren durchaus verschieden von denen in Ägypten und im Vorderen Orient. Wofür also und in welchem Sinn haben die Griechen ihre Standbilder gebraucht? Gegenüber dieser Frage der sozialen Praxis ist die Frage der ästhetischen Produktion sekundär. Dabei wird sich sogar zeigen, dass die soziale Praxis wiederum sehr unmittelbar und viel vitaler auf die künstlerischen Formen wirkt.

\section{HEILIGTÜMER}

Als zum ersten Mal ein Stifter oder eine Stifterin in einem der großen griechischen Heiligtümer wie Delphi, Olympia, Delos, Samos oder auf der Akropolis von Athen ein Standbild von großem Format aufstellte, muss das ein Schritt von weitreichender Wirkung gewesen sein. Das immense Spektrum von Weihgaben, die zum Teil seit Jahrhunderten die Heiligtümer gefüllt hatten, hatte zwei Eigenschaften gemeinsam: Sie waren zum einen grundsätzlich beweglich und zum anderen von begrenzter Dauer. Statuetten aus Bronze und Terrakotta wurden von den Dedikanten in das Heiligtum gebracht, dort wohl auf Tischen, Bänken, Regalen abgelegt und nach einer gewissen Zeit z. T. in ausgedienten Brunnen deponiert. Dasselbe gilt aber auch für die Anatheme von großen Maßen, die seit dem 8. Jahrhundert v. Chr. vor allem in Olympia Eindruck machten, die übermannshohen Dreifußkessel und die Tropaia. Die Dreifüße wurden einfach auf den Boden gestellt, und in dem bekannten Mythos konnte Herakles den delphischen Dreifuß einfach abschleppen; die Tropaia waren an Holzpfählen errichtet, die mit der Zeit verrotteten. Beide Typen von Weihgeschenken wurden nach einiger Zeit abgeräumt und ebenfalls in Brunnen versenkt.

6 s. dazu zuletzt Gunter 2009; Brisart 2011. Für die Kouroi wichtig Kyrieleis 1996. 
Die neuen Standbilder aus Stein dagegen waren unbeweglich, nicht nur wegen ihres Gewichts, sondern weil sie auf einer Basis ortsfest errichtet waren. Zudem waren sie grundsätzlich auf Unvergänglichkeit angelegt. Damit stellten sie nun topographische Fixpunkte dar, und zwar auf Dauer, wie die Tempel, Altäre und andere architektonische Elemente. Man konnte sie nicht entfernen, man konnte sie schwer ignorieren, und man wurde sie nicht wieder los. Es ist deutlich, welch großer Anspruch darin zum Ausdruck kam, ein solches Bildwerk aufzustellen. Da die Orte der Aufstellung die zentralen Heiligtümer der Städte oder gar panhellenische Heiligtümer waren, stellt sich die Frage, wie die Gemeinschaften darauf reagierten. Darüber ist wenig bekannt, aber einige Nachrichten deuten darauf, was ohnehin nahe liegt, dass die Verwaltungen der Heiligtümer mehr und mehr eine Kontrolle ausübten und Regeln entwickelten, um Wildwuchs zu verhindern. Auch in dieser Hinsicht ist Großplastik ein Phänomen der Polis und der interpolitischen Heiligtümer. ${ }^{7}$

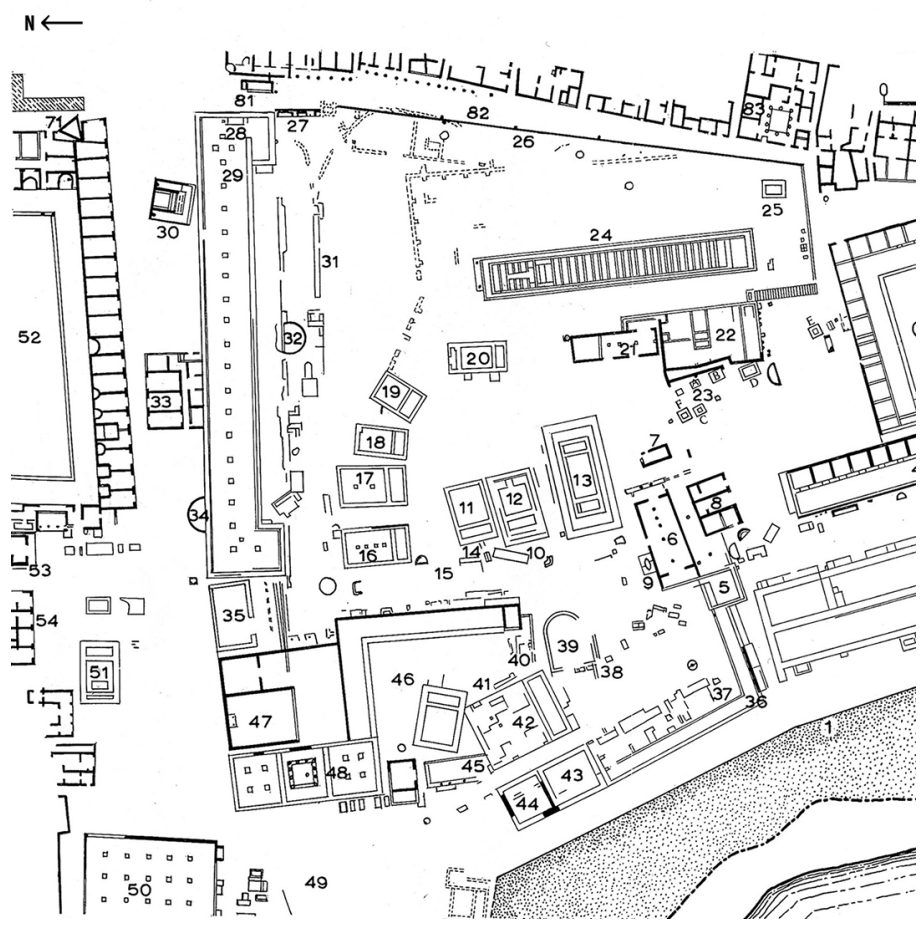

2 Delos, Apollonheiligtum 
Delos. Für die beiden frühesten Zentren großformatiger Skulptur, Kreta und die Kykladen (Abb. 3-8. 20-22), ist die Bedeutung dieser Bildwerke im Rahmen politischer Gemeinschaften von Matteo d'Acunto deutlich gemacht worden. ${ }^{8}$ Im Heiligtum von Delos (Abb. 2) bezeugt die fast zehn Meter hohe kolossale Statue, die die Bewohner der benachbarten Insel Naxos um oder bald nach $600 \mathrm{v}$. Chr. stifteten, ein ungemein anspruchsvolles Auftreten einer Polis (Abb. 4-5): Die Gewinnung der riesenhaften Marmorblöcke, die umfangreichen Bildhauerarbeiten in den Marmorbrüchen und später, der gefährliche Transport über Land durch unwegsames Gelände, die riskante Verschiffung über das Meer und die höchst schwierige Aufrichtung am Zielort bezeugen sehr beträchtliche Aufwendungen einer großen selbstbewussten Gemeinschaft. Das Standbild stellte wahrscheinlich den Gott Apollon dar, der die wenigen kleinen Gebäude im Umkreis von kaum mehr als drei bis vier Metern Höhe weit überragt und den Ort mit seinem weißen Glanz dominiert haben muss. ${ }^{9}$ Lange Zeit war dies das einzige große Bild des Gottes, bis im späteren 6. Jahrhundert v. Chr. ein Tempel mit einem sehr kunstvollen Kultbild errichtet wurde, das in der linken Hand den Bogen, in der rechten eine Gruppe von drei Chariten mit Musikinstrumenten trug (Abb. 7).

Dass Naxos im 7. Jahrhundert v. Chr. eine potente Polis-Gemeinschaft war, zeigt sich nicht nur an der Aussendung von Kolonien nach Sizilien und Nordafrika, sondern auch in der Strukturierung des eigenen Territoriums, mit einem städtischen Zentrum und extraurbanen Heiligtümern für Apollo, Demeter und Dionysos, die das Umland an die Stadt binden. Das stärkste Zeugnis aber ist das Auftreten der Gemeinschaft außerhalb ihrer Stadt, in dem delischen Heiligtum, wo die Naxier offenbar unmittelbar anschließend an den Koloss ein Versammlungshaus, einen oikos, für die gemeinsamen Gelage ihrer Bürger bei den großen Festen errichteten: auch dies das damals bei weitem größte Gebäude am Platz. Damit aber war eine gesamte Neustrukturierung des Heiligtums eingeleitet, denn die Naxier hatten bereits die Statue und den oikos in Hinblick auf eine völlige Neugestaltung dieses Bereichs geplant: Bald darauf errichteten sie ein prächtiges neues Eingangstor und daran anschließend eine Halle, die ebenfalls den Namen der Naxier trugen.

7 Zur Praxis der Aufstellung von Weihgeschenken s. Jacquemin 1999, 101-107; ThesCRA I (2004) $269-281$ bes. $280-281$ s. v. Greek dedications. Introduction, Literary and Epigraphical sources (R. Parker).

8 D'Acunto 2008.

9 Zur technischen Leistung s. Gruben 1997, 267-287; Giuliani 2005. 


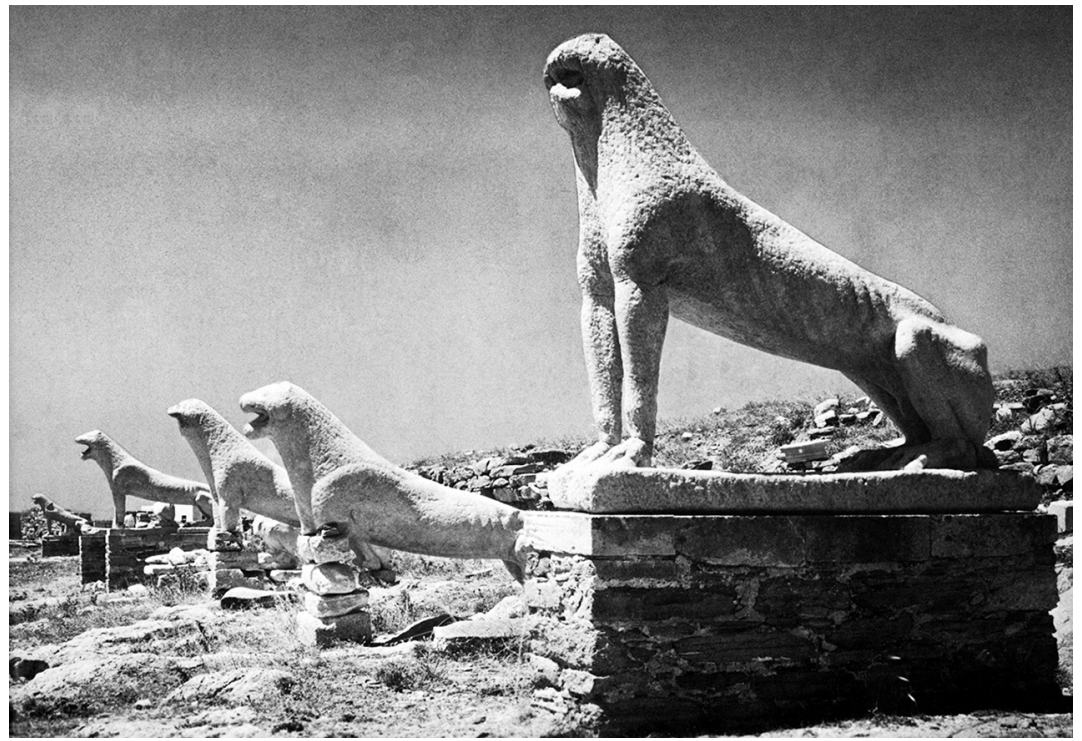

3
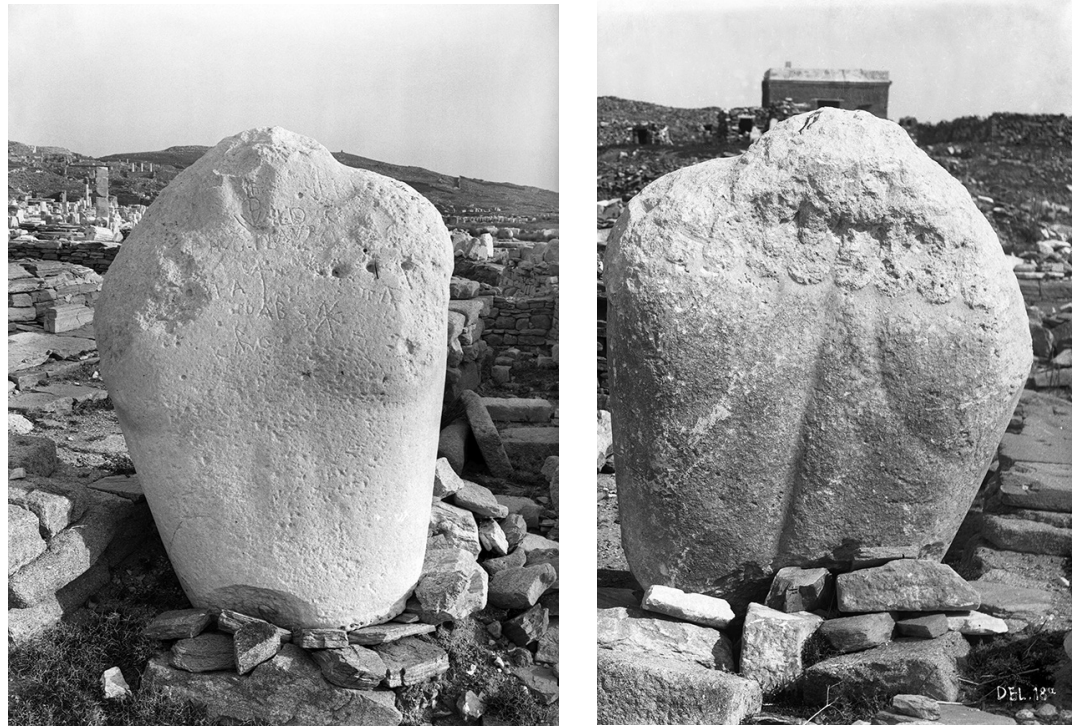

4

5

Delos 3 Löwen-Terrasse, 4-5 Sog. Naxier-Koloss 


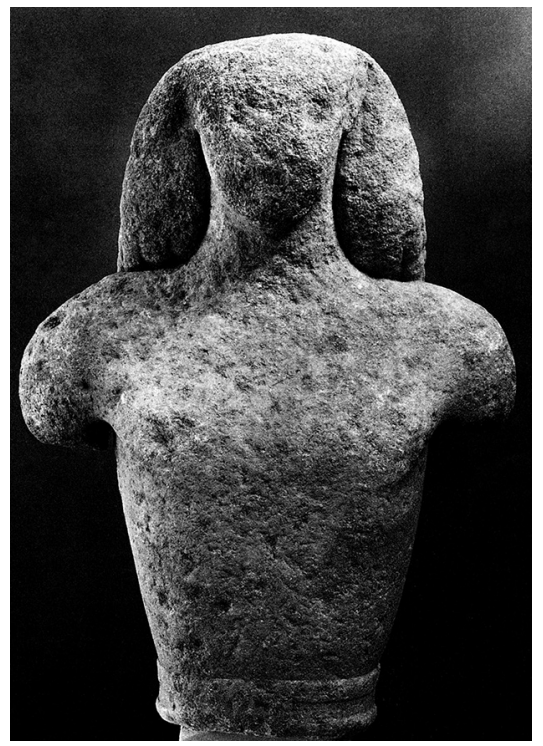

6

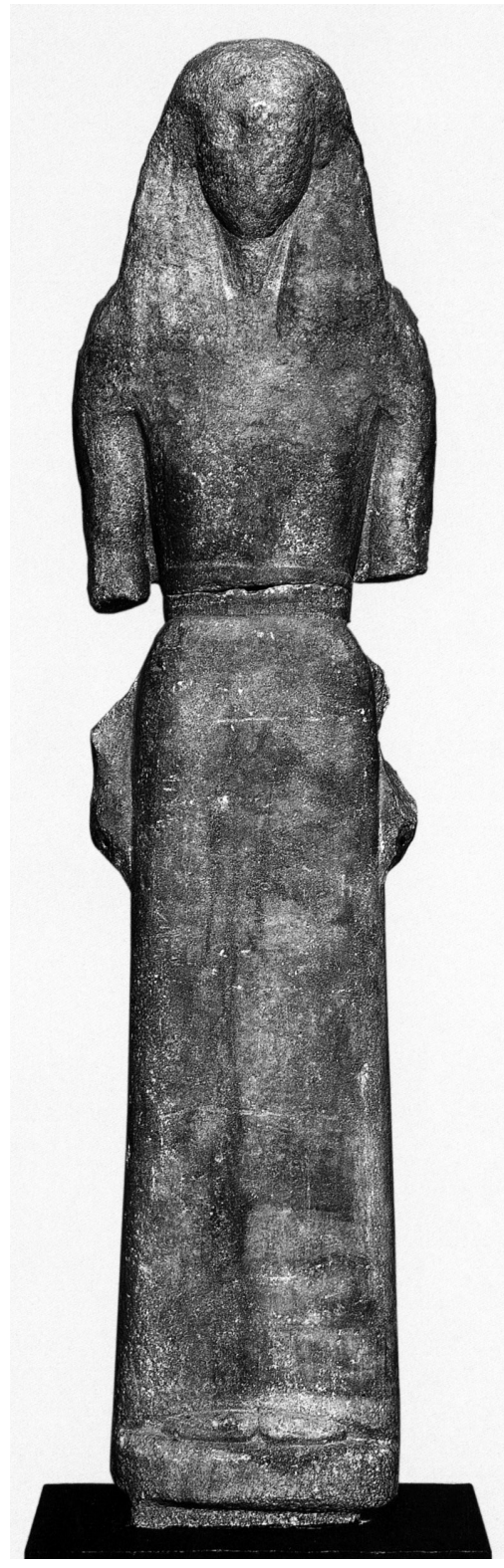

7

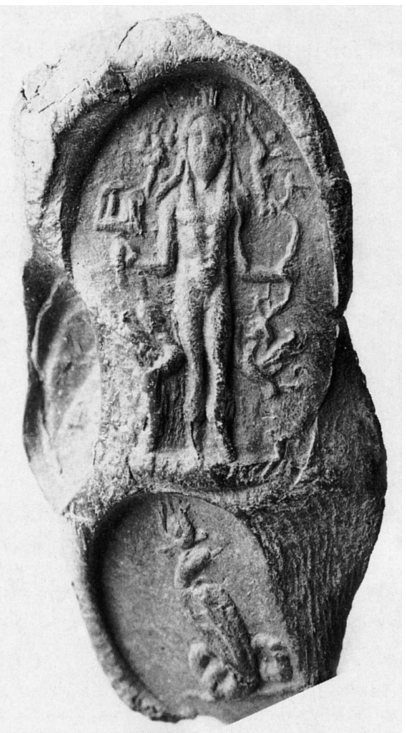

8

Delos 6 Kouros. Delos, Museum, 7 Siegel-Abdruck: Kultbild des Apollon. Delos, Museum, 8 Votivstatue der Nikandre. Athen, Nationalmuseum 
Möglicherweise wurde der Eingang damals überhaupt vom Norden in den Süden verlegt, was dann auch eine Neuordnung des Kultes und seiner Rituale bedeuten würde. ${ }^{10}$ Schließlich muss noch ein weiterer Großauftrag dieser Zeit von einer Polis, offenbar wieder Naxos, ausgegangen sein: die berühmte Löwenterrasse im Norden des Heiligtums (Abb. 3). An der Grenze zur freien Natur waren die Bestien dort auf den Heiligen See mit der Palme ausgerichtet, unter der Leto Apollon geboren hatte. Löwen waren wilde Mächte der Natur, die der Gott unter seine Gewalt gebracht hatte und die er nun als Trabanten zum Schutz des Heiligtums und anderer Räume der menschlichen Ordnung einsetzte. ${ }^{11}$

Das delische Heiligtum gehörte zwar nicht zu Naxos, sondern war ein zentraler Kultplatz der Kykladeninseln, aber Naxos scheint im 7. und 6. Jahrhundert v. Chr. unter den Kykladen eine beherrschende Stellung gehabt zu haben, die es durch Präsenz mit gemeinschaftlichen Bauten und Bildwerken auf Delos zur Geltung brachte. Möglicherweise war das massive Auftreten der politischen Gemeinschaft Naxos und die Neugestaltung des Heiligtums um und nach $600 \mathrm{v}$. Chr. sogar mit einer Neuorganisation des Kults verbunden.12

Früher noch als diese Manifestationen der Polis haben private Stifter aus Naxos Standbilder von großem Format in Delos errichtet. Erstaunlicherweise steht am Anfang der erhaltenen Beispiele eine Frau: Nikandre, die ihr Standbild in das Teil-Heiligtum der Artemis geweiht hat und die sich in der Inschrift ungewöhnlich ausführlich in einer Familie aus Naxos verankert, mit Nennung ihres Vaters Deinodikes, ihres Bruders Deinomenes und ihres Ehemannes Phraxos (Abb. 8). Damit wird offenbar, entsprechend aristokratischer Heiratspolitik, die Verbindung zweier mächtiger Familien angezeigt. Gleichwohl ist es überraschend, dass die Frau sich als »herausragend unter den Frauen« nennt und selbst als Stifterin auftritt, und dass dies auf der interpolitischen Bühne von Delos geschieht: Nikandre präsentiert sich damit zugleich als Vertreterin ihrer Polis. Wenn man bedenkt, dass in späteren Epochen öffentliche Bildnisstatuen für solche Frauen errichtet wurden, die

10 Koloniestädte: D’Acunto 2008, 151. Heiligtümer von Iria und Sangri auf Naxos: Gruben 2001, 367-371. 375-380. Bauprogramm der Naxier auf Delos: Gruben 1997, passim.

11 Bruneau - Ducat 2005, 224-227 Nr. 55. Löwen als Tiere des Apollon, Bezwingung der Löwen und Einsatz als Wächter: Hölscher 1972, 68-99.

12 Naxische >Hegemonie über Delos: D'Acunto 2008, 151-153. 
als Priesterinnen eine öffentliche Stellung erreicht hatten, so kann man fragen, ob auch Nikandre eine Funktion im Kult der Artemis ausübte? ${ }^{13}$

Wenig später setzt die dichtere Reihe der Standbilder junger Männer ein, der Kouroi, wohl idealer Vertreter der männlichen Jugend (Abb. 6). Insgesamt neun Exemplare stammen aus dem späteren 7. und frühen 6. Jahrhundert v. Chr. Sie sind ebenfalls aus naxischem Marmor gearbeitet und wurden nach ihrem Stil meist zuversichtlich ebenfalls Stiftern aus Naxos zugewiesen. Vielleicht sollte man nicht ganz so direkt aus dem Material und Stil auf die Auftraggeber schließen; denn in dieser Frühzeit werden noch nicht alle Inseln eigene Marmorbrüche und Bildhauerwerkstätten besessen haben, so dass auch Stifter von anderen Orten ihre Aufträge nach Naxos gegeben haben können. Wichtig wäre die Frage vor allem unter dem Aspekt, ob hier führende Personen von verschiedenen Inseln miteinander in Konkurrenz treten oder ob auf Naxos bereits eine dichtere Elite entstanden ist, die diese Praxis der Bildweihung ausgebildet hat. Deutlich ist aber, dass gleichzeitig mit den gemeinschaftlichen Manifestationen der Polis Naxos auch individuelle Stifter von dieser Insel - und vielleicht von anderen Orten - in einem gemeinsamen Heiligtum außerhalb des eigenen Polisgebietes in individueller Initiative auftritt. ${ }^{14}$

Zweifellos war dabei viel Konkurrenz im Spiel - dabei ist auffallend und aufschlussreich, wie homogen die Ausdrucksformen des Wettstreits sind: Kouroi und Koren werden zu fast kanonischen Symbolen des sozialen Anspruchs. Um das zu verstehen, muss man sich klar machen, dass Konkurrenz immer in zwei Richtungen gehen kann: entweder Distinktion oder Konvergenz. Wenn mein Nachbar mit einem Porsche auftrumpft, kann ich mich entweder mit einem Jaguar von ihm absetzen oder ebenfalls einen Porsche aufbieten, vielleicht ein neueres Modell. Wenn die archaischen Eliten Griechenlands in ihrem Kampf um Rang und Prestige mehr auf relative Homogenität der Ausdrucksformen setzten,

$13 \mathrm{Zu}$ den frühen Weihungen snaxischer Standbilder in Delos s. D'Acunto 2008. Zur Nikandre als Zeugnis aristokratischer Heiratsverbindungen s. Vorster 2002, 98-103. Die Frage, ob die Statue die Stifterin oder die Göttin Artemis darstellt, ist nach wie vor umstritten. Das eingestiftete Attribut in der linken Hand war m. E. eher ein Kranz als ein Bogen mit Pfeil, die wohl prononcierter nach vorne gehalten würden.

$14 \mathrm{Zu}$ den Kouroi von Delos s. Meyer - Brüggemann 2007, 174-181 Nr. 193225; Zuweisungen an Naxos: Kokkorou-Alewras 1995, 81-85 Nr. 5-8. 12. 12a; 103-108 Nr. 61-62. 64. 65-67. 
so ist das also nicht im Sinne sozialer Harmonie zu deuten. Vielmehr bezeugen sie damit in der Konkurrenz die zunehmende Verdichtung der Bürgergemeinschaften, offenbar seit dem späteren 7. Jahrhundert v. Chr. Denn >kompetitiv<, im Sinn des Wettstreits miteinander, und `soziak, im Sinn von gemeinschaftlich, gehen hier eng zusammen.

In diesem Sinn sind die kollektiven Bau- und Bilddenkmäler der Polis und die individuellen Weihungen der Eliten zwei Aspekte derselben politischen Strukturen. In neueren Arbeiten wird zum Teil die Vorstellung vertreten, Weihungen der Polis und der individuellen Mitglieder der Oberschicht stünden in einer grundsätzlichen Antithese, in der ein fundamentaler Konflikt zwischen `Elite` und `Mittelklasse` ausgetragen werde..$^{15}$ Dies Modell ist wohl in dieser einfachen Weise kaum zutreffend: Auf der Akropolis von Athen wie im Heraion von Samos setzen die gemeinschaftlichen Bauprojekte und die dichten Reihen der Statuenweihungen zur selben Zeit ein und entwickeln sich parallel zueinander. Offensichtlich waren es nicht gegensätzliche politische Gruppen, die sich in kollektiven und in individuellen Manifestationen präsentierten. Beide Formen der Selbstdarstellung gehen zusammen. ${ }^{16}$

Mit dem Anspruch der öffentlichen Repräsentation in Bildwerken wuchs auch das Ansehen derer, die die Standbilder herstellten: der Bildhauer. Ebenfalls aus Delos stammt die Basis eines überlebensgroßen Kouros, der von einem Bildhauer Euthykartides gearbeitet, geweiht und stolz signiert ist. An den Ecken ist sie bewehrt von Köpfen eines Löwen, eines Widders und einer Gorgo, die als Mächte der Natur in den Dienst des Menschen genommen sind. Solche, nicht ganz seltenen, Eigenweihungen von Bildhauern sind nicht, wie man z. T. liest, Zeugnisse für die soziale Emanzipation von Arbeit als solcher; sondern alle Bekundungen von handwerklichem Stolz kommen von Männern, die spezifische Beiträge zur Kultur der Elite geleistet haben. Die arbeitsteilige und spezialisierte Produktion der Elite-Kultur führte zu einer starken Differenzierung des Prestiges unter den arbeitenden Schichten. Die oberen

15 Die grundsätzliche Antithese zwischen »elitist « und "middling « Ideologien wurde bekanntlich von Morris 1996 entwickelt. Fokussierung und Anwendung auf Anatheme in Heiligtümern bei Neer 2001. In der deutschsprachigen Forschung wurden diese wichtigen Ansätze kaum wahrgenommen. $16 \mathrm{Zu}$ Athen und Samos s. Franssen 2011. Meinen eigenen Standpunkt gegenüber I. Morris und R. Neer kann ich hier nur knapp skizzieren. 
Gruppen der Produzenten, allen voran die Bildhauer, konnten in ihren anspruchsvollen Votiven neben die Mitglieder der Oberschicht treten. ${ }^{17}$

Zusammen gesehen, ergänzen sich die früharchaischen Bildwerke von Delos zu einem Kosmos von Gottheit, Menschen und Tieren, der, wie noch zu zeigen ist, der konzeptionellen Ordnung der frühen Polis entspricht.

Delphi, Samos, Athen. Die für Delos nur hypothetische Vermutung, dass die Praxis der Weihung von Standbildern in großem Format durch Neuordnungen von Kulten befördert wurde, wird durch die Entwicklung in anderen Heiligtümern bestärkt. In Delphi hat das Heiligtum des Apollon im frühen 6. Jahrhundert v. Chr. eine Art Neugründung erfahren. Im so genannten 'Ersten Heiligen Krieg، hatte die Amphiktyonie, eine mächtige Koalition von >umliegenden` Mächten, insbesondere Thessalien, Athen und Sikyon, das Heiligtum gegen Übergriffe der nahe gelegenen Stadt Kirrha verteidigt und im Jahr 586 v. Chr. die panhellenischen Spiele dort neu organisiert. Dies war offenbar der Anlass dafür, dass unmittelbar danach mehrere mächtige Poleis dort höchst ambitionierte statuarische Denkmäler stifteten (Abb. 9).

Naxos, das sich damals mit dem kolossalen Apollon in Delos hervortat, errichtete wenig später in Delphi eine über 2 Meter hohe Sphinx auf einer fast 10 Meter hohen Säule, die ihrerseits auf einem Felsklotz hoch über dem archaischen Zugang zu dem Heiligtum wachte (Abb. 10-11). ${ }^{18}$

Zur selben Zeit muss die Stadt Argos eine von Herodot erwähnte Statuengruppe der Brüder Kleobis und Biton nach Delphi geweiht haben, die ihre Mutter, die Priesterin, im Wagen in das weit entfernte Hera-Heiligtum gezogen hatten und dafür mit einem glücklichen Tod belohnt wurden (Abb. 12). Die Identifizierung der beiden bekannten Statuen mit diesem Denkmal ist zuletzt mehrfach in Zweifel gezogen worden, jedoch mit wenig plausiblen Gründen: Die Signatur eines Bildhauers aus Argos in Delphi und die ganz ungewöhnlichen Zwillingsstatuen passen so genau zu Herodots Überlieferung, dass es ein unwahrscheinlicher Zufall wäre, wenn das überlieferte Denkmal verloren wäre und stattdessen ein zweites Denkmal erhalten wäre, das eben so gut zu der Überlieferung passt. Mit ihren ungewöhnlich kräftigen gedrungenen Körperformen re-

$17 \mathrm{Zu}$ Weihgeschenken von Bildhauern und Töpfern s. Scheibler 1979. Die hier skizzierte Deutung muss später begründet werden; kurze Zusammenfassung bei Hölscher 2007, 49.

18 Picard 1991, 31-33. Der Eingang zum Heiligtum lag damals offenbar im Süden, unterhalb des Felsens, auf dem die Naxier-Sphinx stand. 


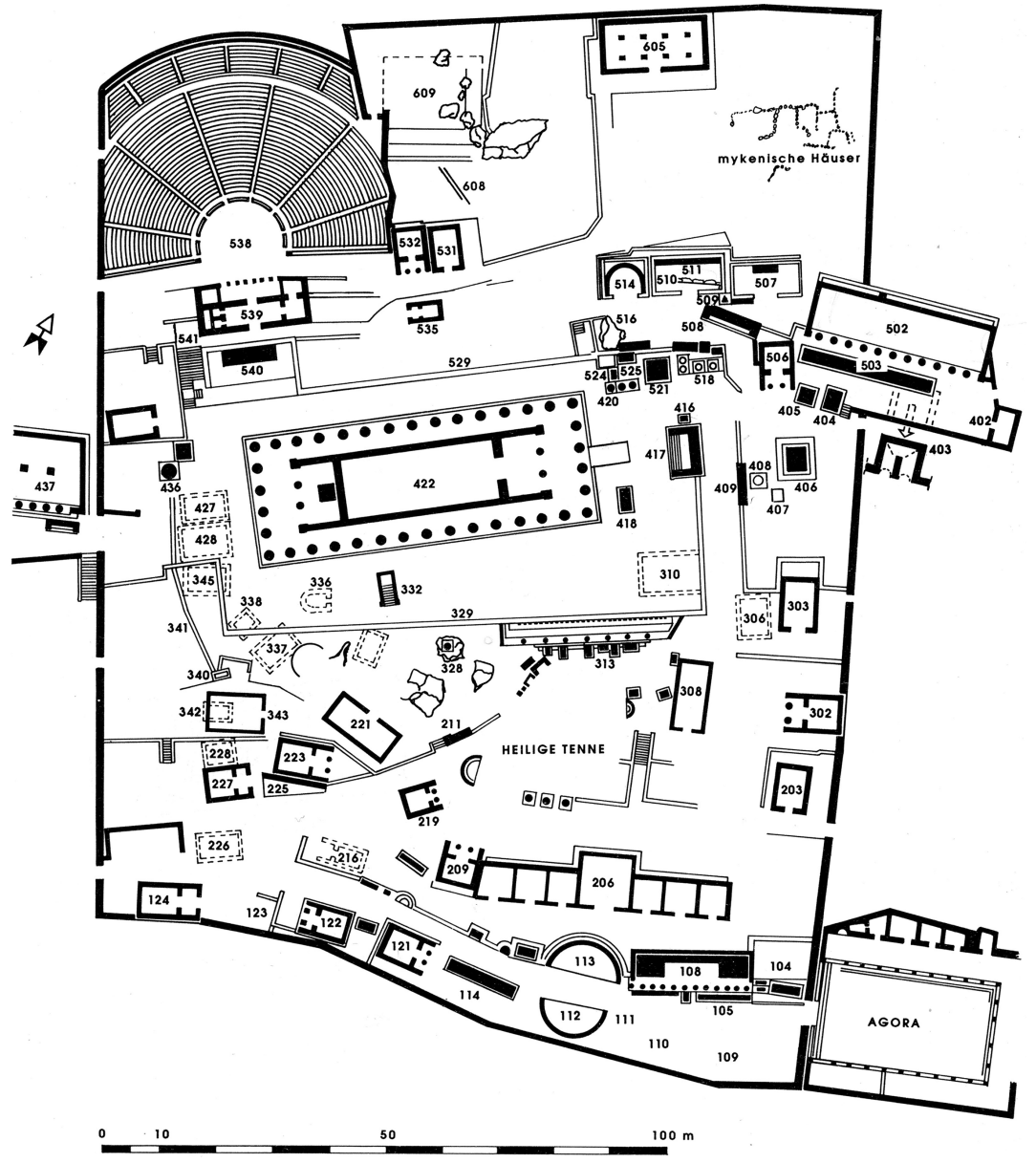

9

Delphi 9 Apollonheiligtum 

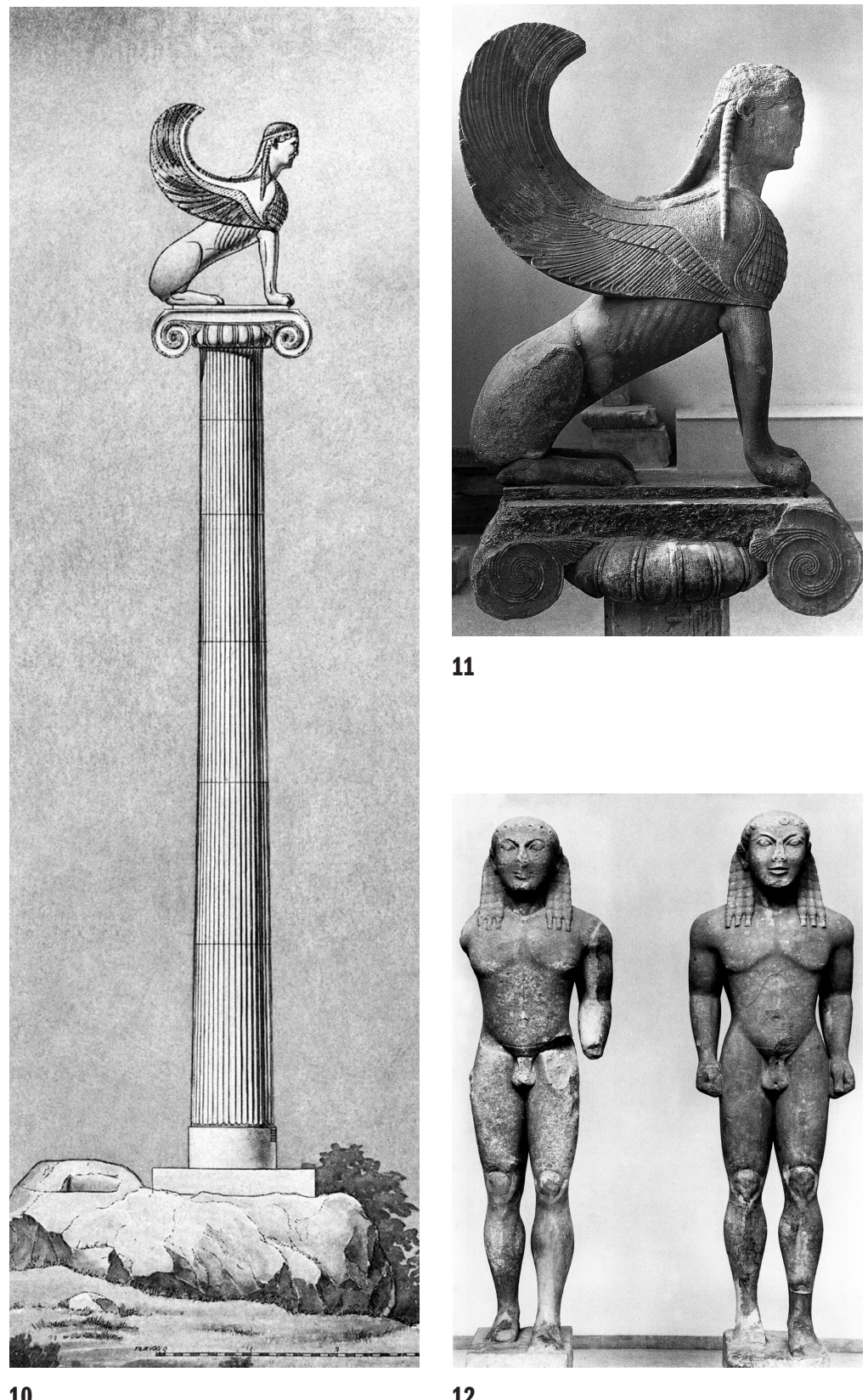

11

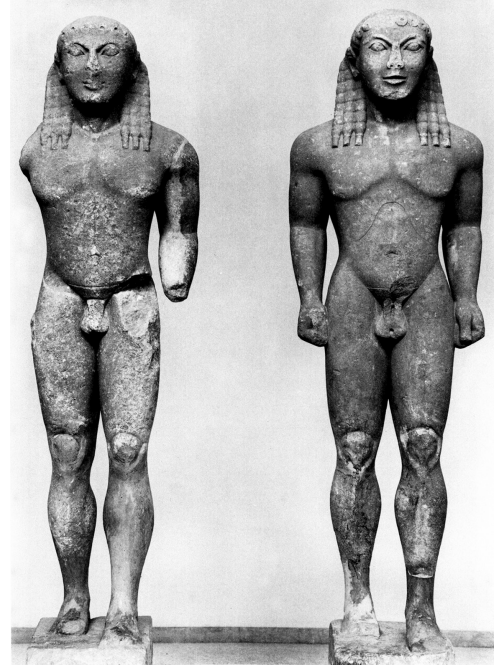

12

Delphi 10 Sphinx der Naxier. Rekonstruktion, 11 Sphinx der Naxier. Delphi, Museum, 12 Standbilder des Kleobis und Biton. Delphi, Museum 


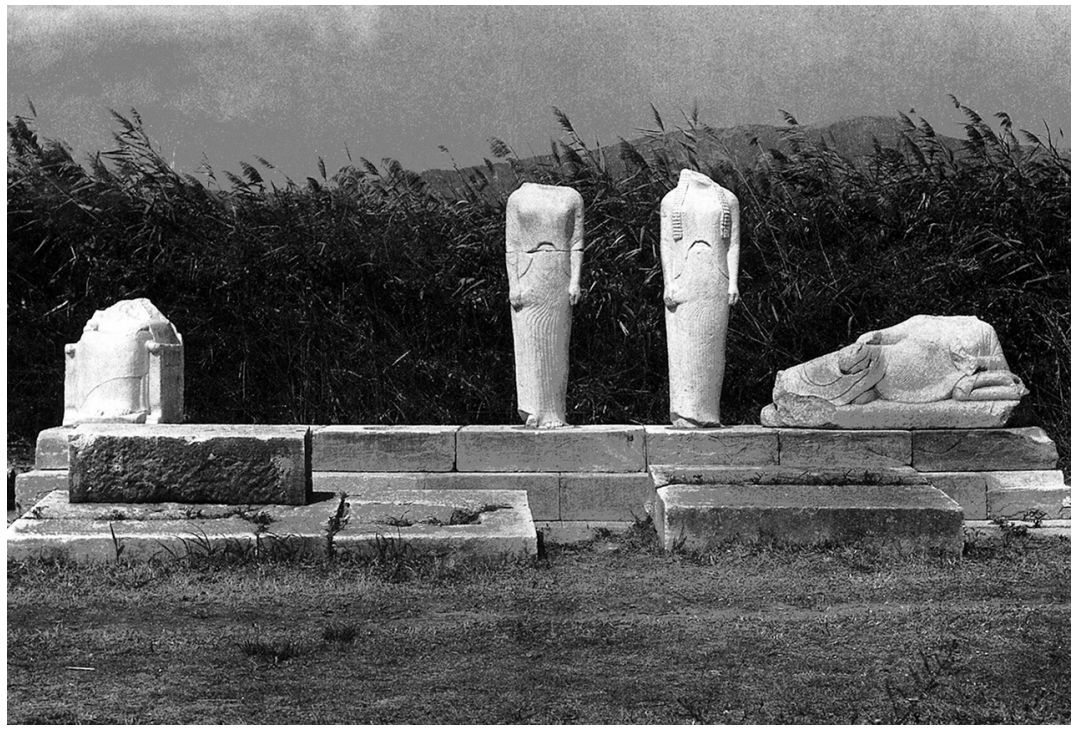

13

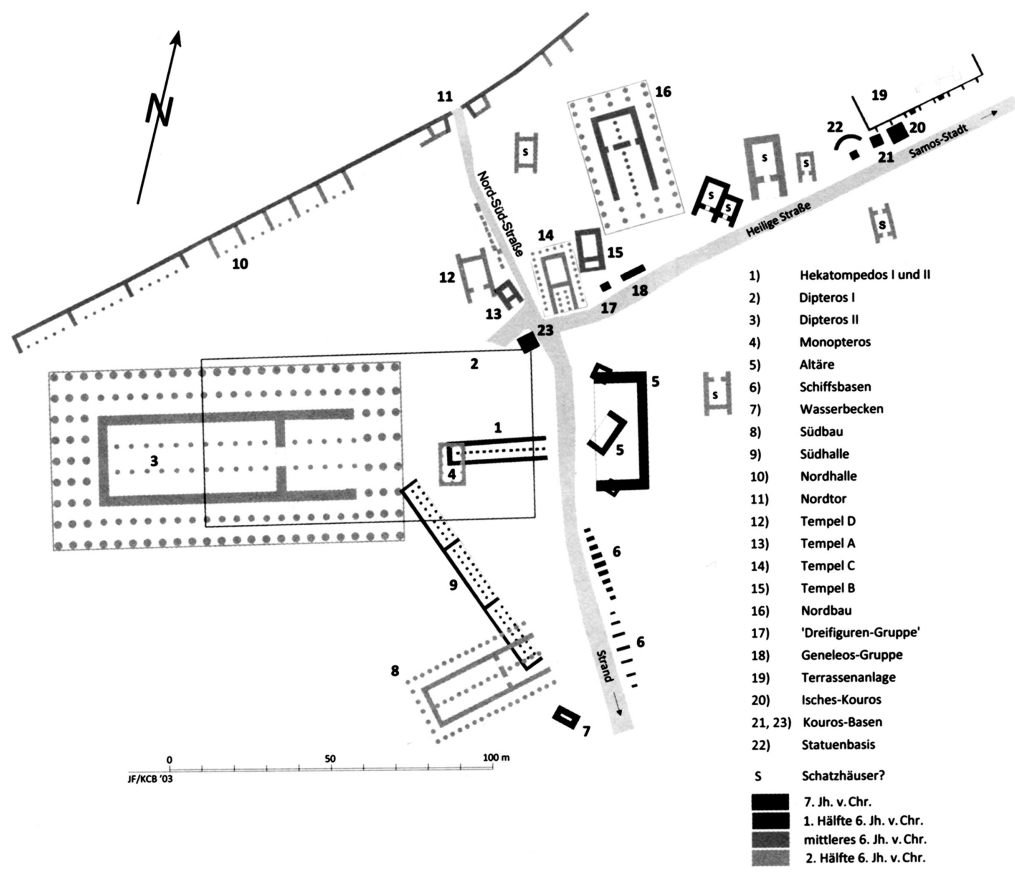

14

Samos 13 Familiendenkmal des -ilarches, 14 Samos Heraheiligtum 


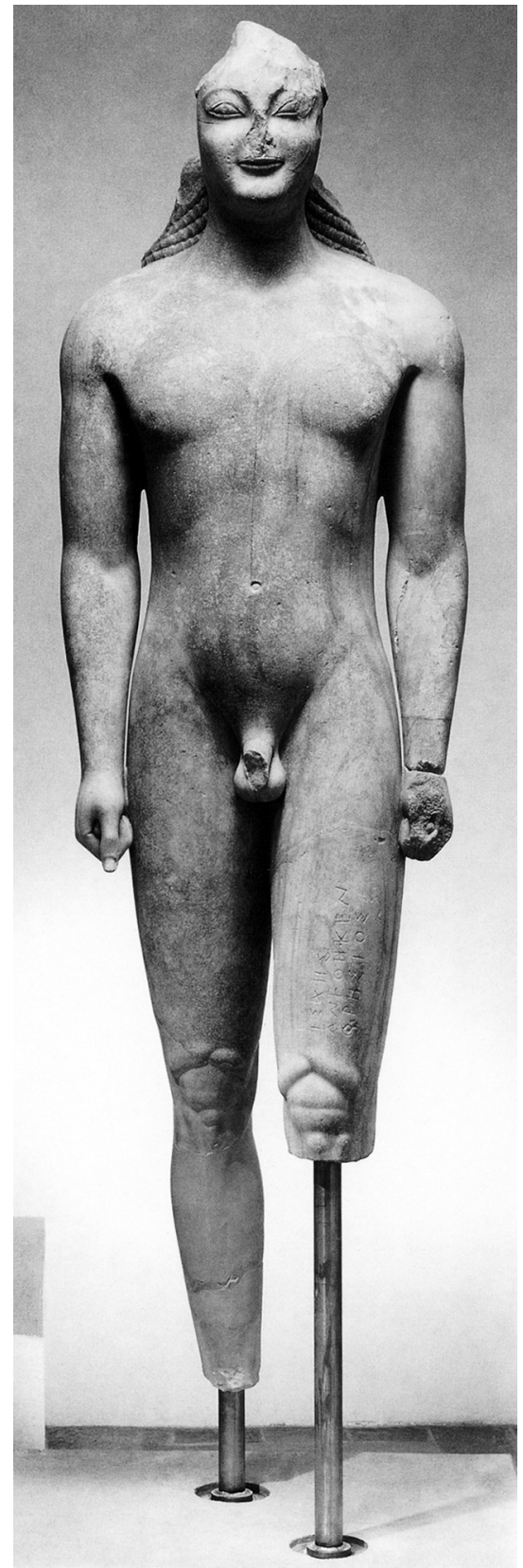

15

Samos 15 Standbild des Isches. Samos, Museum 


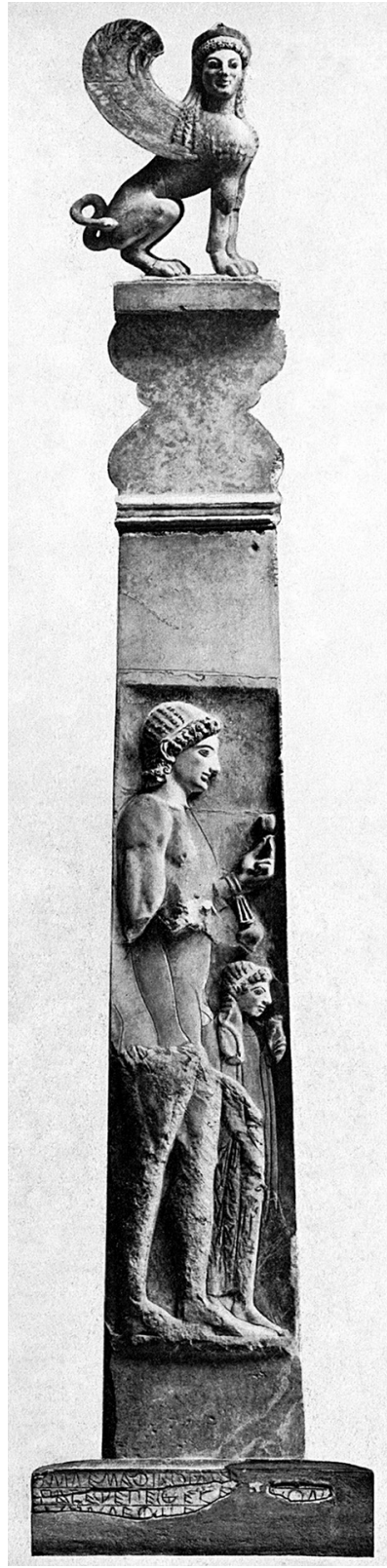

16

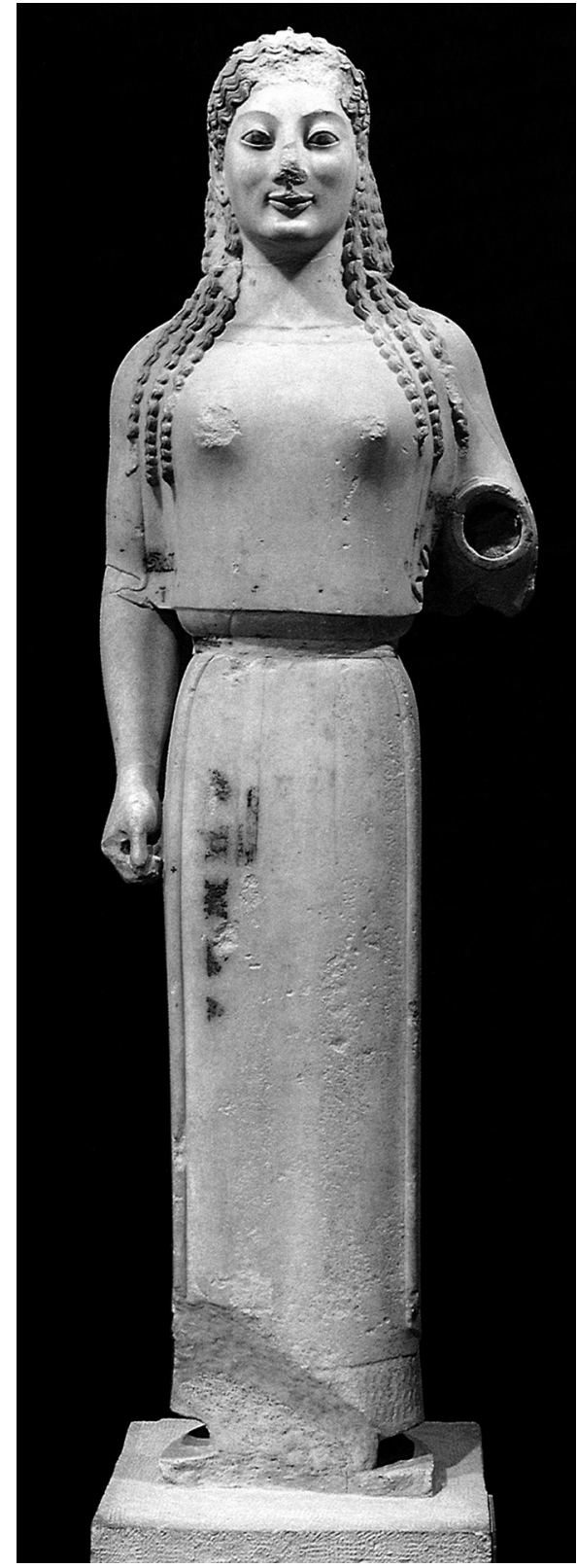

17

Athen 16 Grabstele zweier Geschwister. New York, Metropolitan Museum, 17 Sog. Peplos-Kore. Athen, Akropolis-Museum 


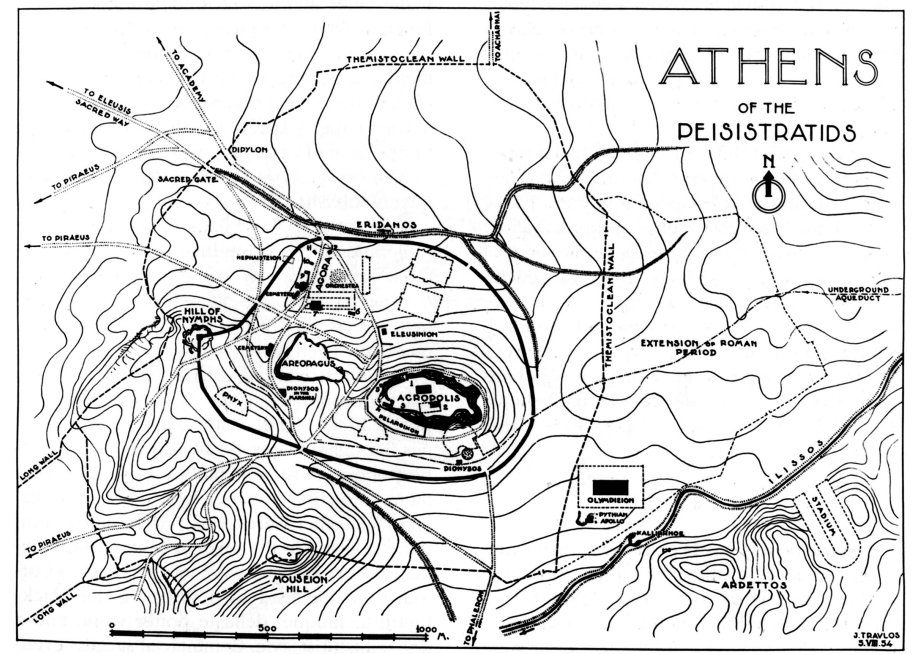

18

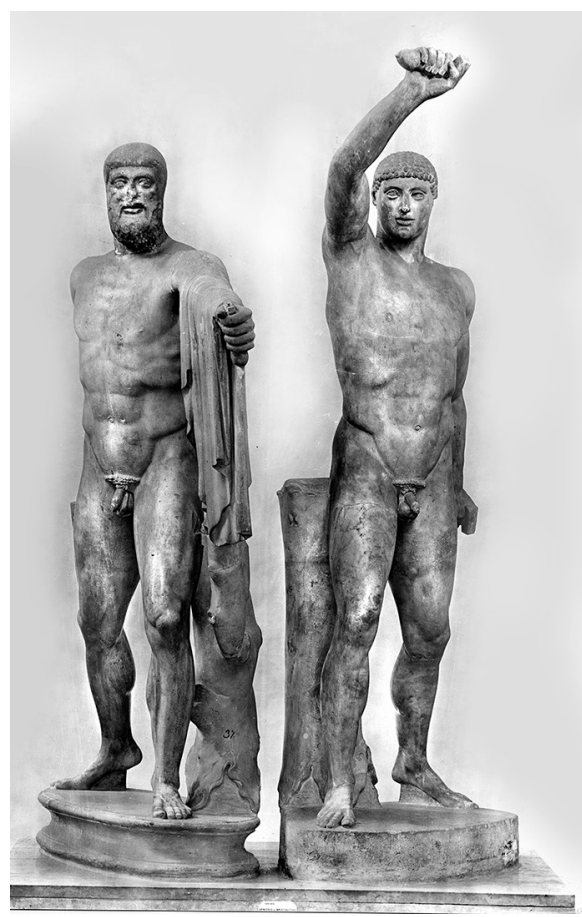

19

Athen 18 Athen in archaischer Zeit, 19 Statuen der Tyrannenmörder. Rekonstruktion 
präsentieren die Brüder ihre Stadt Argos als Ort von körperlicher und ethischer Exzellenz. ${ }^{19}$

Diese und andere Mächte dieser Zeit nutzten die Bühne des neu begründeten Heiligtums, um ein kompetitives Konzert um Ansehen und Macht anzustimmen. Damit bestätigten und stärkten sie zugleich die interpolitische Kohärenz der griechischen Poliswelt.

Auch in Delphi schließen sich wieder das Monster der Sphinx, die idealen Jünglinge aus Argos und das im Tempel vorauszusetzende Bild des Apollon zu einem Spektrum aus Gottheit, Menschen und Tieren zusammen. Die Bilderwelt dieser Zeit war so weitgehend in sich geschlossen, dass sich auch bei verschiedenen Stiftern ein relativ homogener Kosmos von Bildthemen ergab.

Ähnlich wie im panhellenischen Delphi hat in den Poleis Samos und Athen eine Neuorganisation der Haupt-Heiligtümer und eine gleichzeitige Erneuerung der Kultordnung zu einem Boom von statuarischen Weihungen geführt. In Samos wurde im frühen 6. Jahrhundert v. Chr. der gesamte Zugang zum Heiligtum neu gestaltet: Statt der Fahrt zu Schiff, die im Süden des Heraions endete, wurde ein Prozessionsweg zu Land eingerichtet, der bei einem Eingang im Osten ankam (Abb. 14). Damit muss eine stärkere Beteiligung und Kohärenz der Bürgerschaft verbunden gewesen sein, die sich jetzt in einer geschlossenen Prozession formierte. Kurz danach wurde der erste riesige Dipteros-Tempel begonnen. In dieser Phase fängt auch die dichte Reihe der statuarischen Weihungen an. Sie beginnen mit kolossalen Figuren wie dem Kouros Isches (Abb. 15) und führen im 2. Viertel zu sehr aufwändigen Manifestationen führender Familien, wie dem Anathem des -ilarches, mit Vater, Mutter und vier Kindern (Abb. 13), oder den mindestens vier Koren, die ein gewisser Cheramyes errichtete..$^{20}$

In Athen auf der Akropolis sind die Zusammenhänge noch deutlicher: 566 v. Chr. wurden bekanntlich die Panathenäen neu begründet: wie der Name sagt, als Fest aller Athener. Wahrscheinlich zu diesem Zeitpunkt wurde ein bereits vorher begonnener monumentaler Tempel mit reichem Skulpturenschmuck geweiht, gleichzeitig beginnt die unvergleichlich dichte Reihe der Weihungen von Koren (Abb. 17). Aus den Inschriften lässt sich ersehen, dass das Zentralheiligtum Athens nun ein Referenzpunkt für Weihungen aus ganz Attika wurde: So wurde

19 Hdt. 1, 31. Picard 1991, 33-36. Neuere Diskussion der Benennung bei Bumke 2004, 59-69.

20 Dazu Franssen 2011. 
eine Weihung von zwei Brüdern Aristion und Lysias gestiftet, deren Grabreliefs in Ost-Attika gefunden wurden. Die Formen einer frühen Kore in rein samischem Stil machen deutlich, dass sofort Bildhauer von auswärts kamen, um den neuen Bedarf zu erfüllen. ${ }^{21}$

An beiden Orten aber, in Samos wie in Athen, steht es außer Zweifel, dass diese ganze Entwicklung bereits vor der Etablierung der Tyrannis, vor Peisistratos und vor Polykrates, einsetzte, d. h., dass sie von der aristokratischen Oberschicht getragen wurde. ${ }^{22}$

Die wichtigste Erkenntnis aus Samos liegt aber darin, dass hier die Topographie der Standbilder erfasst werden kann. Isches, die Familie des -ilarches und eine ganze Reihe von weiteren Basen reihen sich entlang der `Heiligen Straße،, vom Eingang zum Vorplatz des Tempels; weitere Basen finden sich um den Altar. In diesen Gebieten wurden auch die meisten der archaischen Skulpturen gefunden, die offenbar nicht weit verschleppt worden sind. Das heißt, dass die Standbilder eng auf die kultischen Rituale bezogen waren, die im Heiligtum vollzogen wurden: Sie stellten ein ideales Publikum für die von der Stadt eintreffende Prozession und die Opferhandlungen am Altar dar.

Kreta. Was uns weitgehend fehlt, sind die Kultstatuen in den Tempeln. Seit der Zeit um 600 v. Chr. gibt es in der ganzen griechischen Welt einen Schub an monumentalen Tempelbauten, von Sizilien über Korfu, Athen, die Inseln bis Kleinasien. In all diesen Tempeln muss es Kultbilder gegeben haben, von denen wir uns fast durchweg allenfalls allgemeine Vorstellungen machen können. Als frühe Beispiele des beginnenden 7. Jahrhunderts v. Chr., eigentlich noch vor der Phase der Großplastik, können die bekannten Götterbilder aus Bronzeblech über Holzkernen aus einem Tempel in Dreros/Kreta dienen, die dort auf einer Bank vor der Rückwand der Cella gestanden hatten (Abb. 20). Dabei muss man grundsätzlich von zwei Arten von Kultbildern ausgehen: Die einen, im Allgemeinen früheren Bilder waren klein, aus leichteren Materialien wie Holz und Metallblech gefertigt, und darum im Kultritual

21 Zusammenfassend Franssen 2011, bes. 242-252. Weihung von Aristion und Lysias: Kissas 2000, 255-256 Nr. 16-17; deren Grabreliefs, aus Velanideza: Richter 1961, Nr. 67. 70.

22 Die Vorstellung, dass monumentale archaische Tempel vornehmlich von Tyrannen errichtet seien, ist ein alter Topos der Forschung. Für Athen wird das nicht nur durch die Chronologie des Tempels auf der Akropolis widerlegt, sondern auch durch den ersten Tempel des Zeus, Vorgänger des Olympieion, mit acht Säulen in der Front, um 580 v. Chr. datiert. 


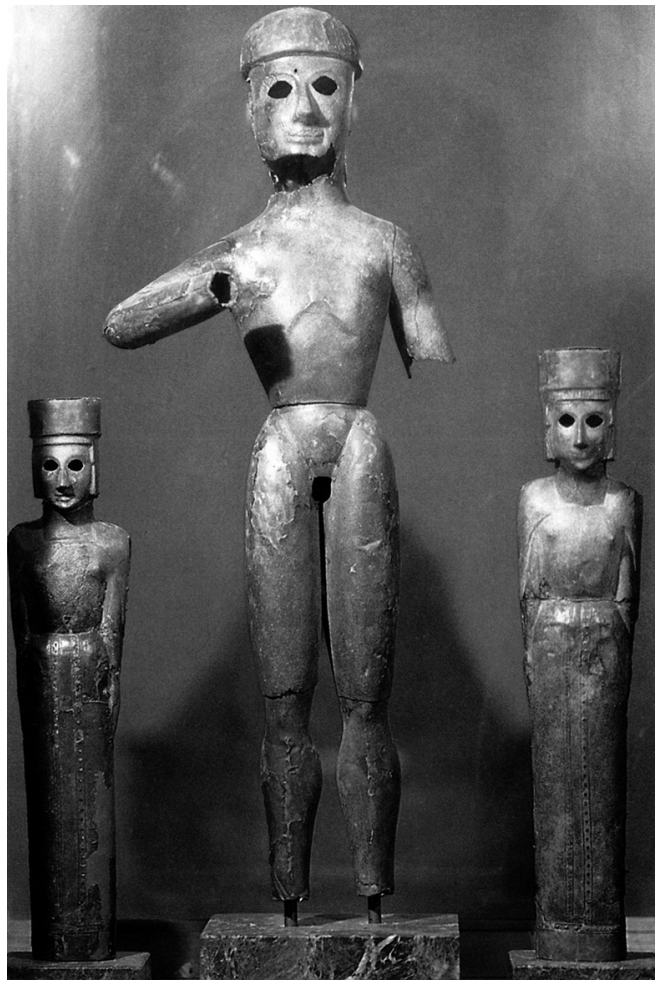

20

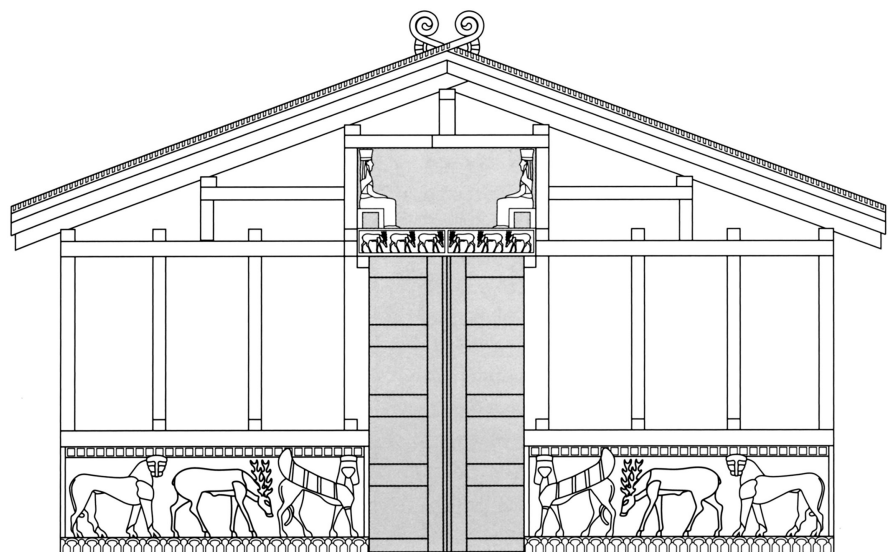

21

Kreta 20 Dreros, Tempelbilder aus Bronzeblech. Heraklion, Museum, 21 Tempel von Prinias. Rekonstruktion 


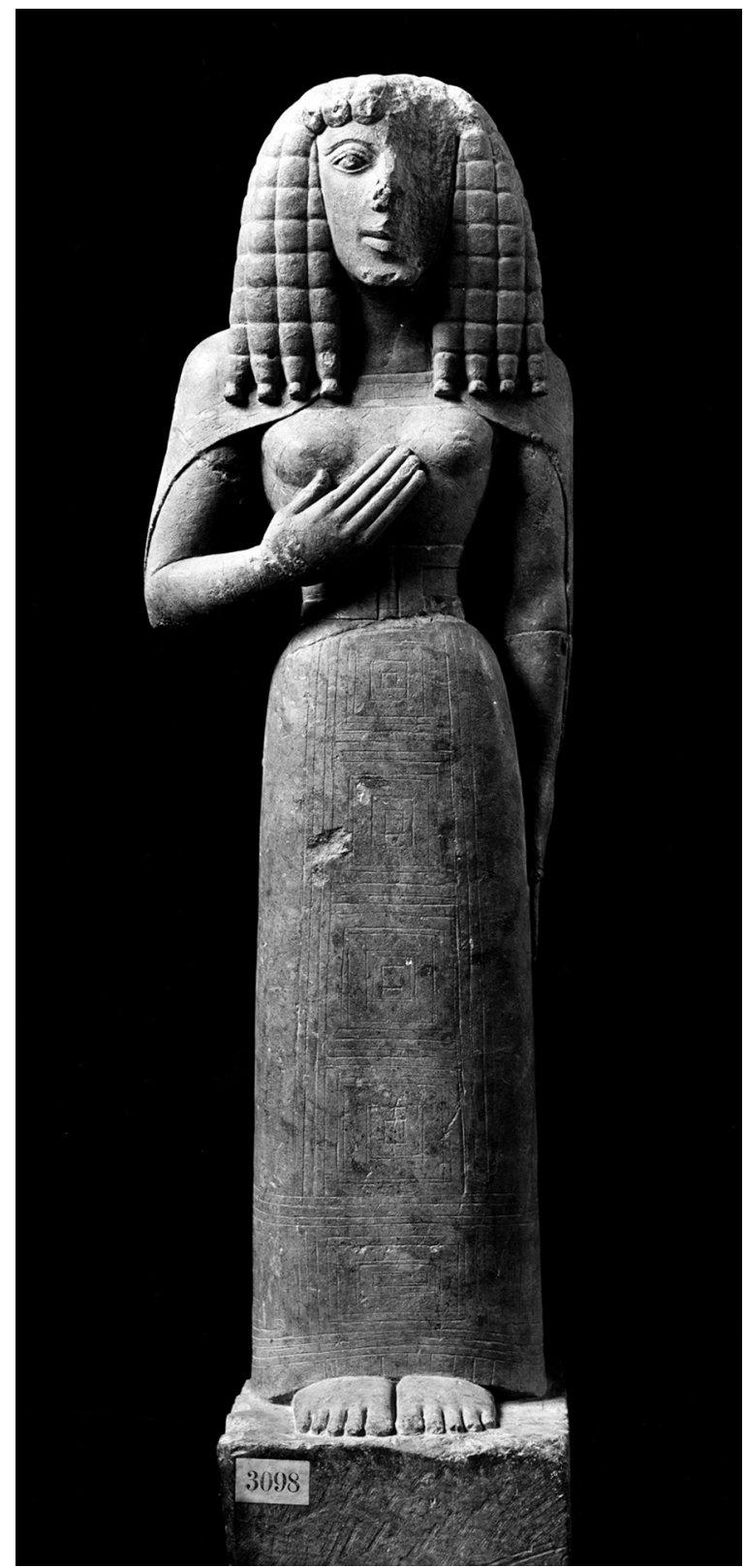

22

Kreta 22 Kore, sog. Göttin von Auxerre. Paris, Louvre 
beweglich: zum Herumtragen in Prozessionen, Waschen im Fluss, See oder Meer, Salben, Einkleiden. Die anderen waren von großem Format, zunehmend aus Stein gemeißelt oder in Bronze gegossen, ortsfest und daher weniger leicht $\mathrm{zu}$ ınutzen`. Beide Arten aber waren Bilder, in denen die Gottheit beim Kult konkret präsent war. ${ }^{23}$

Zugleich werden auf Kreta die ersten Beispiele von figürlichem Schmuck an Tempelbauten greifbar. In Prinias ist die architektonische Ordnung des typischen griechischen Tempels noch nicht ausgebildet, aber an der Fassade wird eine ungewöhnlich reiche Bilderwelt entfaltet, die sich zu einem System zusammenfügt (Abb. 21). Zu Seiten des Eingangs standen auf Sockelreliefs zwei Sphingen, Monster der imaginierten Wildnis, als Wächterinnen. Wahrscheinlich schlossen auf beiden Seiten Sockelfriese mit Zügen von bewaffneten Reitern an, die die männliche Elite in der Sphäre außerhalb der Stadt repräsentieren; dagegen erschienen auf den Innenwänden der Türe, dem >Haus`zugehörig, Mädchen in ritueller Nacktheit. Der Türsturz nahm das Thema der wilden Natur wieder auf, mit drohenden >Panthern ‘ auf der Außenund zahmen Hirschen auf der Innenseite. Auch hier steht die Wildnis im Dienst der menschlichen Kultur: Auf der Unterseite des Sturzes finden sich stehende bekleidete Frauen, während darüber rundplastische Frauen thronen. Die hierarchische Steigerung der weiblichen Gestalten führt wohl von Kultdienerinnen über vornehme Frauen bis zu göttlichen Wesen, die wegen ihrer Mehrzahl wahrscheinlich niedrige Göttinnen sind. Am wahrscheinlichsten ist eine Deutung als Chariten oder Horen, untergeordnet dem Gott oder der Göttin des Tempels, etwa Apollon oder Artemis. Vieles bleibt dabei unsicher, aber deutlich ist, dass hier ein ganzer Kosmos entfaltet ist, der von der wilden Natur über die menschliche Ordnung bis zu den niedrigeren und höheren Gottheiten reicht. Es ist der Kosmos einer frühen Polis mit einer Elite von Rittern. Seit dem 6. Jahrhundert v. Chr. wurden dann ähnliche komplexe Bild-Ordnungen für den Schmuck kanonischer Tempel mit Metopen, Friesen und Giebeln konzipiert, in Korfu, Athen und andernorts. ${ }^{24}$

23 Früharchaische Skulpturen aus Kreta: Brisart 2011, bes. 273-314. Zu frühen Kultbildern s. Romano 1980; ThesCRA IV (2004) 52-65 s. v. Kultbilder (F. Hölscher); Zu Dreros: ThesCRA IV (2004) 56 f. Nr. 14 s. v. Kultbilder (F. Hölscher).

24 Zu Prinias s. D’Acunto 1995; Brisart 2011, 277-281. 


\section{DIE PRÄSENZ DER BILDWERKE IN HEILIGTÜMERN, AN GRÄBERN UND AUF ÖFFENTLICHEN PLÄTZEN}

Das führt auf die allgemeinere Frage, welche Gestalten und Themen mit den Bildwerken in den Lebensräumen der frühen Polis-Kultur präsent gemacht wurden. Damit wird der Kreis über die Heiligtümer hinaus erweitert.

In den Heiligtümern waren es zunächst die Götter, die in ihren Bildern >anwesend gemacht wurden, meist im `Haus` des Tempels. Es war, wie wir gesehen haben, eine sehr konkret gemeinte Anwesenheit: zum Vollzug von rituellen Handlungen mit der Gottheit, wie Waschen, Ankleiden, Bewirtung, oder für die Gottheit, wie Opfer, Singen von Hymnen, Tanzen, athletische Agone.

Auf die Bilder der Götter antworteten, meist in den sakralen Bezirken um den Tempel aufgestellt, die Bilder von Menschen, vor allem die Kouroi und Koren. Deren Bedeutung wird immer wieder als problematisch angesehen, weil die Bildwerke meist anonym bleiben und wenig spezifische Indizien für ihre Deutung geben. Dabei ist sicher, dass sie in der Regel weder die Stifter noch die Gottheit, an die die Stiftung sich richtet, darstellen. Dagegen sprechen die vielen Koren, die Stiftungen von männlichen Dedikanten sind, und die vielen Fälle, in denen Koren für männliche und Kouroi für weibliche Gottheiten gestiftet wurden. Ein Indiz für die Deutung sind zunächst die kleinformatigen Kouroi und Koren aus Bronze und Terrakotta: Sie können nur anonyme, typische Bilder sein, die ein angemessenes Geschenk für die Gottheit darstellten. Es liegt darum nahe, auch die großformatigen Standbilder in der überwiegenden Mehrzahl als anonyme Vertreter der jungen Generation der gegenwärtigen Gesellschaft zu verstehen. Bestätigt wird diese Deutung durch die wenigen Figuren in großem Format, die durch Inschriften benannt sind, wie die des Familiendenkmals aus Samos: der Vater -ilarches wie zum Symposion gelagert, die Mutter Phileia thronend, dazu die Töchter Ornithe, Philippe und ein weiteres Mädchen als Koren sowie ein Sohn als Kouros: also Mitglieder der Gesellschaft der Lebenden, die aus dem Kollektiv ihrer Standesgenossen durch individuelle Namen herausgehoben sind. Entsprechend wurden etwa in Olympia die Standbilder der Sieger, im Typus des Kouros, individuell ausgezeichnet. Die kollektive Darstellung anonymer Kouroi und Koren aber hat ihre Begründung im Kult: Die Polisgemeinschaften formierten sich im tatsächlichen Ritual der Götterkulte vor allem in Chören und Tänzen ihrer jungen Männer und Mädchen, die die Zukunft der Gemeinschaft verkörperten und im 
gemeinsamen Auftreten die größte Freude für die Götter darstellten. Die vornehmen Stifter errichteten den Göttern Figuren dieser Altersgruppen als Geschenke. Im Lauf der Zeit wuchsen diese Standbilder in den Heiligtümern zu dichten Bild-Gemeinschaften zusammen. ${ }^{25}$

In dieser dauerhaften Gestalt stellen die Standfiguren eine Bildgemeinschaft im Heiligtum dar, die einen Bedeutungsrahmen für die Handlungen der Gemeinschaft der Lebenden herstellt. In Samos ist es noch nachzuvollziehen, wie die Bildwerke vor allem die >Heilige Straße vom Eingang zum Tempel säumten und den Vorplatz des Tempels mit dem Altar umgaben. Ähnlich wurden in Olympia die ersten Siegerstatuen im Zentrum des Kultes am Ziel der archaischen Rennbahn errichtet, danach an den rituellen Wegen innerhalb des Heiligtums. In Epidauros wird der rituelle Raum um den Altar von den Basen der hellenistischen Ehrenstatuen eingefasst und definiert. Überall wurden die Rituale in Gegenwart und im Angesicht einer wachsenden Zahl von bildlichen Mitgliedern der Gemeinschaft vollzogen, die den lebenden Vertretern die akzeptierten sozialen Leitbilder vor Augen stellten. ${ }^{26}$

Der menschlichen Gemeinschaft wiederum wird die Gegenwelt der wilden Natur gegenübergestellt, mit Sphingen, Löwen, Gorgonen und anderen Monstern. Sie stellen jene Mächte dar, gegen die die Gemeinschaft der menschlichen Kultur sich behaupten muss, und die die Götter und Menschen bezwingen, um sie zum Schutz der eigenen Lebensordnung gegen böse Mächte einzusetzen: als Wächter über die sakralen Bezirke und insbesondere die kostbaren Tempelbauten. ${ }^{27}$

In entsprechender Weise wurden an den Gräbern die Toten mit ihren Standbildern und Reliefstelen in die Gemeinschaft der Lebenden einbezogen. Auch hier wurden sie dem Schutz jener Naturkräfte, vor allem Sphingen und Löwen, unterstellt (Abb. 16). Das Spektrum der Darstellungen ist breiter als in den Votiven der Heiligtümer, aber wieder dominieren die Kouroi, daneben die Koren, nun durchgehend durch Namen individualisiert. Hier ist es besonders erstaunlich, dass nicht die Tradition der Genealogie durch mächtige Familienoberhäupter betont wird; allgemein wird wohl die Bedeutung genealogischer Ideo-

25 Neuere Diskussion der Deutungen bei Meyer - Brüggemann 2007, 1529. 121-130; Franssen 2011, 94-101. 101-105. Die hier vertretene Deutung folgt im wesentlichen Schneider 1975.

26 Dazu Hölscher 2002.

27 Hölscher 1972, 68-99; Tiere und Wildnis: Hölscher 1999, 17-20; Winkler-Horacek 2000. 
logie in der Polis vielfach überschätzt. Tatsächlich geht es auch an den Gräbern wieder vor allem um die Jugend: Nach den Epigrammen wurden Grabdenkmäler besonders für jung Verstorbene errichtet, die der Tod vor Erreichen der Blüte des Lebens ereilt hatte und bei denen, gegen den Lauf der Natur, die Eltern die Kinder begraben mussten. So wie in den Heiligtümern der Glanz der jungen Generation als größtes Potential der Stadt gerühmt wird, wird an den Gräbern der Verlust in dieser Altersgruppe in besonderem Maß beklagt. Es ist ein Verlust für die Gemeinschaft. ${ }^{28}$

Dem entsprechen die Orte der Gräber und ihrer Bilder. Jene Familien, die starke Präsenz im städtischen Zentrum der Politik anstrebten, legten ihre Grabbezirke an den Zugangs- und Ausfallstraßen der Städte an. Dort wuchsen die Grabmonumente im Lauf der Zeit zu einer idealen Präsenz zusammen, die anzeigte, welche Familien in der Stadt das Sagen hatten. Andere Familien dagegen traten mit Grabstatuen im Rahmen ihrer Landgüter in der chora auf: So hatte eine mächtige Familie, vielleicht die Alkmeoniden, ihre Grabstätte in Attika in der Nähe von Anavyssos, auch hier offenbar an einer größeren Landstraße, die entlang der Küste nach Athen führte. Hier bezeichneten die Figuren wohl nicht so sehr den konkreten Landbesitz, den man besser mit Grenzsteinen bezeichnete, sondern den Machtanspruch gegenüber den Gefolgschaften, die lokal konzentriert waren. ${ }^{29}$

An ihren Orten, die durchweg weite Sichtbarkeit garantierten, sprachen die Grabfiguren mit ihren Epigrammen einen kollektiven Kreis von nicht eingegrenzten Betrachtern an: Bleib stehen und klage über den Tod des Dargestellten, wie schön er war - und doch sterben musste! ${ }^{30}$

Erst relativ spät wurde der dritte öffentliche Raum, die Agora als Ort für Bildwerke erschlossen. Den Anfang machen zwei Athletenstatuen, für den Pankratiasten Arrichion von Phigalia auf der Agora seiner Heimatstadt und für den Boxer Kreugas von Epidamnos im Heiligtum des Apollon Lykeios an der Agora von Argos. Beide waren im Wettkampf unter Bruch der Regeln zu Tode gekommen und post mortem zu Siegern in den Spielen von Olympia bzw. Nemea erklärt worden; ihre außergewöhnliche Ehrung auf der Agora ist offenbar damit begründet,

28 Archaische Grabstatuen: Richter 1961. Epigramme: Kissas 2000, 37-80. 247-258.

29 Archaische Grabmäler Athen und Attika: D’Onofrio 1982. Anavyssos: Neer 2010, 21-30 (dort Deutung als Markierung von Landbesitz).

30 s. das Epigramm zum Grabmal des Kleoitas: Kissas 2000, 249 Nr. 4. 
dass ihr Tod in besonderem Maß als ruhmvoll und darum als politische Angelegenheit angesehen wurde. Die Statue des Arrichion wird von Pausanias erstaunlich genau beschrieben, im Typus eines Kouros. Beide Standbilder sollten offenbar den Mitbürgern im Zentrum des öffentlichen Lebens als Vorbilder des ehrenvollen Verhaltens vor Augen stehen. ${ }^{31}$ In Athen wurde dann nach dem Sturz der Tyrannen der entscheidende Schritt zu dem ersten eigentlich politischen Denkmal getan: der Statuengruppe der Tyrannenmörder (Abb. 19), mit der durch Volksbeschluss eine politische Tat als Gründungsakt der neuen demokratischen Staatsform geehrt, und die als Leitbild für politisches Verhalten aller athenischen Bürger errichtet wurde. ${ }^{32}$

Durch die ganze Antike wurden die politischen Räume mit Bildwerken von politischem Charakter ausgestattet. Lykurg ist stolz darauf, dass in Athen auf der Agora keine Statuen von Athleten, sondern nur von Staatsmännern stehen, und Vitruv mockiert sich über die Bewohner von Alabanda, dass sie auf ihrer Agora Standbilder von Athleten und im Gymnasion solche von Rechtsgelehrten stehen hätten. Das ist in zweierlei Hinsicht interessant: zum einen, weil es zeigt, dass es thematische Standards gab, zum anderen, weil es darauf hinweist, dass diese Standards nicht fixiert waren; denn auch die Leute von Alabanda hatten natürlich ihre Konzepte, sie definierten nur die Öffentlichkeit ihrer Agora anders als Lykurg und Vitruv. ${ }^{33}$

Bildwerke gehören in soziale Räume mit deren sozialen Praktiken. Dabei ist das Verhältnis von Bildwerken und Räumen reziprok: Einerseits bedingen die Räume, welche Bilder in ihnen aufgestellt werden. Andererseits ist dies Spektrum nicht kanonisch fixiert, sondern kulturell variabel: Die Bewohner von Athen und von Alabanda gaben ihrer Agora durch Aufstellung von Bildwerken einen unterschiedlichen Charakter. Insofern prägen die Bilder umgekehrt auch den Charakter der Räume. ${ }^{34}$

31 Paus. 6, 18, 7.

32 Brunnsåker 1953.

33 Lykurg. Leokrates 51; Vitr. 7, 5, 6.

34 Hölscher 2012. 


\section{DIE PRAKTIKEN DES PRÄSENTMACHENS}

Wenn wir von der >Aufstellung sprechen, so bezeichnen wir damit den technischen Akt. Auch 'Stiftung، oder 'Weihung sind ungenau und betreffen nur Teilaspekte. Am ehesten wird man der Bedeutung der Vorgänge gerecht, wenn man sie im Rahmen des archaischen Austausches von Geschenken versteht. Eine Votivstatue in einem Heiligtum oder ein Standbild auf einem Grab ist grundsätzlich ein Ehrengeschenk, $\gamma \varepsilon \rho a \varsigma$, für die Gottheit bzw. für die oder den Verstorbenen. Geschenke sind reziprok, aber grundsätzlich kein unmittelbarer Gegenwert für eine Leistung des Partners. Votivgeschenke an eine Gottheit sind zwar oft als Dank für einen Erfolg oder Sieg dargebracht worden, aber eine derart unmittelbare Erwiderung ist nicht die einzige Möglichkeit: Geschenke können zu vielen anderen Anlässen gemacht werden. Geschenke verleihen dem Empfänger >Ansehen`, $\tau \mu \eta\rceil$; sie erhöhen auch das Ansehen des Schenkenden. Allgemein dienen sie der Herstellung eines dauerhaften Verhältnisses der gegenseitigen Gunst, in dem die Partner einander ethisch verpflichtet sind und sich in allfälligen Situationen auf die Gunst und Hilfe des Partners verlassen können.

Diese Praxis ist im gesellschaftlichen Leben, das heißt zwischen menschlichen Partnern, bei Homer vielfach bezeugt. Sie gilt auch für das Verhältnis der Menschen zu den Göttern und den Toten. Mit dem Geschenk einer Kore oder eines Kouros an die Gottheit erfüllt man keine Schuld durch ein Wert-Äquivalent, sondern erweist mit einem Wertstück, $\alpha \gamma \alpha \lambda \mu \alpha$, eine Freude und Ehre, die dem tatsächlichen Auftreten eines schönen Mädchens oder jungen Mannes im Heiligtum zu Ehren der Gottheit entspricht. Dasselbe gilt für Stand- oder Reliefbilder auf Gräbern. Auch sie sind nicht obligatorisch den Verstorbenen geschuldet, sondern eine freiwillige Gabe an solche Tote, gegenüber denen besondere Zuwendung angemessen schien.

Die Motive und Themen dieser bildlichen Geschenk-Praxis änderten sich nach den wechselnden kulturellen Vorgaben der betreffenden Gesellschaften, nach den Rollen und Verhaltensweisen in den skonzeptuellen Gemeinschaften der Menschen, Götter und Toten. Darin liegt ihre Geschichtlichkeit begründet. Die Geschichte der antiken Votive als ısozialer Austausch zwischen den verschiedenen Gruppen dieser Gemeinschaften ist eine künftige Aufgabe der Forschung. 
Die Qualitäten, die in den Standbildern zum Ausdruck gebracht werden, sind kollektive Leitbilder und haben insofern stark kommunikativen und öffentlichen Charakter. Bei den jungen Männern ist der nackte Körper das Distinktiv jener physischen Leistungskraft und Attraktion, die in den athletischen Agonen zur Grundqualität griechischer Männer ausgebildet wurde. Dass die Nacktheit der Athleten nicht etwa ein Relikt kultureller Primitivität war, wussten die Griechen selbst, denn sie überlieferten, dass sie erst im späten 8 . Jahrhundert v. Chr. eingeführt wurde, also gerade zu der Zeit, als die entstehende Polis anscheinend erste Formen der Ausbildung der jungen Männer etablierte und ihr mit der Einrichtung von öffentlichen Spielen eine institutionelle Form gab. Seither wurde der nackte männliche Körper zum Grundthema der griechischen Kunst und Kultur, weit über den funktionalen Bereich der Athletik hinaus. ${ }^{35}$ Bei den Mädchen waren es die reichen und prächtigen Gewänder, die die Körper umspielten und artikulierten, die eine ähnliche Wirkung ausübten. Dabei wurde mit dem leichten Anheben des Gewandes über den Füßen eine Geste zum Leitmotiv gemacht, die zugleich Eleganz der Haltung und potentielles Schreiten suggerierte und dazu das Faltenspiel des Stoffes raffiniert bereicherte. Bei jungen Männern wie Mädchen aber bestechen die Köpfe zunehmend durch kunstvoll perlendes Haar und vor allem durch das gewinnende Lächeln. ${ }^{36}$

Die Grundvoraussetzung für diese eminente Geltung physischer Qualitäten sind die Strukturen der griechischen Polisgesellschaften. Es gab keine dauerhaften Positionen der Macht, die den sozialen Rang einer Person als festen `Besitz` gesichert hätten; darum gewann die unmittelbare Wirkung der Person in der Präsenz von Wort und Erscheinung eine immense Bedeutung. Alle gemeinschaftlichen Angelegenheiten wurden im direkten Miteinander ausgehandelt. In dieser >Kultur des unmittelbaren Handelns` wurde Anmut, Charis, zu einer öffentlichen, politischen Qualität, in der sinnliche und ethische Werte in eins gesetzt wurden. ${ }^{37}$

Damit wird ein Phänomen angesprochen, das m. E. von Anbeginn für die ganze griechische Bildkunst konstitutiv ist: ihr emphatischer sinnlicher Realismus. Darüber ist sich die neuere Forschung jedoch

35 Zum nackten Körper in der griechischen Kultur und Kunst s. Himmelmann 1990; Hölscher 2003; Daehner 2005; Hurwit 2007.

$36 \mathrm{Zu}$ den Mädchen s. Schneider 1975; Meyer - Brüggemann 2007, 27-29. 37 Meier 1985. 
alles andere als einig. Neuere Ansätze, vor allem kulturanthropologischer Prägung, sehen die früharchaische griechische Bildkunst als grundsätzlich unrealistisch an. Der Ausgangspunkt für diese Auffassung ist die Tatsache, dass in der Frühzeit Grabstatuen und Götterbilder als shma, `Zeichen<, benannt werden, so wie auch unfigürliche Pfeiler und Stelen als `Zeichen auf Gräber gestellt wurden. Diese `Zeichenhaftigkeit sei in frühen Kultbildern in der Form von roh behauenen Steinen oder primitiv geschnitzten Hölzern zu erkennen, wie sie in späteren Schriftquellen beschrieben werden. Bilder des Dionysos, die aus einem Pfahl mit aufgesetzter Maske gefertigt waren, oder des Hermes, die aus einem Pfeiler mit Kopf und Phallus bestanden, werden als Relikte dieser frühen Auffassung des Bildes als `Zeichen` der Gottheit verstanden. Daraus sei zu erkennen, dass Ikonizität kein ursprüngliches Ziel der griechischen Bildkunst gewesen sei, und aus dieser Voraussetzung sei allgemein die starke Stilisierung früher griechischer Skulpturen zu erklären, die in ihrer Vierkantigkeit noch von dem ursprünglichen Pfeiler der >Bilder ' von Göttern und Toten geprägt sei. Erst die Bildkunst des 5. Jahrhunderts v. Chr. führe zu einer >naturalistischen`Wiedergabe. ${ }^{38}$

Der geistesgeschichtliche Hintergrund dieser Auffassung ist das Bestreben der Nachkriegszeit, der griechischen Kultur eine fremdartige Urtümlichkeit zu bewahren und sie dabei von dem Vorwurf der >Klassizität` zu befreien. Sofern es dabei allgemein darum geht, die Griechen als $>$ Fremde $<$ zu sehen, wird man gerne zustimmen: Die hier dargestellte >Bildpraxis` ist von dem neuzeitlichen >musealen Habitus des Umgangs mit `Kunst` weit entfernt. Doch mit der Auffassung griechischer Bildwerke als unrealistische Zeichen ist m.E. der Charakter der frühen Bildwerke grundsätzlich verkannt. Die Benennung eines Bildwerks als `Zeichen ist keine fundamentale Definition, sondern eine Bezeichnung einer Qualität. Sie kann nicht bedeuten, dass das Bild als solches nichts anderes als ein Zeichen ist - so wenig wie etwa die Bezeichnung einer wohltätigen Person als `Symbolk von Menschenliebe

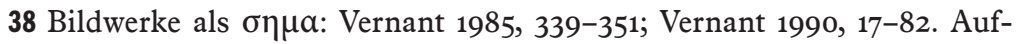
gegriffen und produktiv weitergeführt vor allem in der angelsächsischen Forschung: zuletzt Neer 2011, 14-19; besonders prägnant Neer 2012, 111-116, bes. 115: "The very concept of realism - of a statue that resembles a real person - is alien to Greece in this period«. Eine angemessene Würdigung und Diskussion dieser bedeutenden und herausfordernden Konzepte, die in Deutschland wenig wahrgenommen wurden, kann an dieser Stelle nicht geleistet werden. 
oder eines Wissenschaftlers als \Symbol von Gelehrsamkeit bedeutet, dass es sich nicht um konkrete Personen, sondern um Symbole handelt. Wenn ein Bild ein shma einer Person genannt wird, so wird damit zunächst gesagt, dass es sich nicht um die Person selbst handelt, sondern um eine Darstellung in einem anderen Medium.

Auf welche Weise die Beziehung zu der Person hergestellt wird, ist damit nicht gesagt; jedenfalls ist damit nicht ausgeschlossen, dass es mit visueller Ähnlichkeit geschieht. Dies ist das Wesen des >Bildes«: dass es in konkreter Visualität in der konkreten Lebenswelt für eine Person, einen Vorgang oder einen Gegenstand eintritt.

Die Vorstellung, dass griechische Götterbilder aus anikonischen Pfeilern und Steinen oder primitiv geschnitzten Holzplanken entstanden seien, ist von A. A. Donohue stringent widerlegt worden. ${ }^{39} \mathrm{Bei}$ den erhaltenen früharchaischen Bildwerken aber ist der Eindruck >unrealistischer Stilisierung ein anachronistisches Urteil nach den Maßstäben späterer >realistischer Kunst. Die archaische griechische Kunst ist durch und durch von dem unbändigen Willen geprägt, von Generation zu Generation immer mehr >Realität $<$ in die Bildwerke einzuholen. Ein Epigramm zu der (nicht erhaltenen) Grabstatue eines adeligen Verstorbenen in der Gestalt eines jungen Mannes, eines Kouros, bringt diese Intention in aller Deutlichkeit zum Ausdruck: »Schau das Denkmal des Kleoitas an und klage, wie schön er war und doch sterben musste«. Wie immer das Bild formal gestaltet und stilisiert war, jedenfalls sollte es die körperliche Erscheinung dieses Toten sinnlich vor Augen stellen. In demselben Sinn sind auch die frühen Bildwerke des 8. und 7. Jahrhunderts v. Chr. intentional >realistisch. Bereits im 5. Jahrhundert v. Chr. wurden die frühen Standbilder, die man dem Urkünstler Daidalos zuschrieb, als so >lebendig`angesehen, dass man sagte, sie müssten gefesselt werden, damit sie nicht davonliefen.

>Realität aber ist kein objektiver Befund, sondern abhängig von kulturellen Vorgaben. Jede Epoche, und so auch die archaische Zeit Griechenlands, hat eigene Vorstellungen von Realität. Das 5. Jahrhundert v. Chr. führte in der Bildkunst nicht erstmals zu >Realismus und 'Naturalismus‘, sondern entwickelte neue Konzepte von Realität. Die griechische Bildkunst hat damit einen Weg eingeschlagen, der zwar weniger urtümlich ist, dafür aber im Lauf der Geschichte zu dem

39 Donohue 1988; Hölscher 2004, 53. 
nicht weniger unheimlichen Unternehmen immer weiterer instabiler Realismen verführt hat.

Nur unter der Voraussetzung eines derartigen >konzeptionellen Realismus k konnten die Bildwerke ihre soziale Funktion erfüllen und den konkreten >Umgang der lebenden Menschen mit den Göttern, den toten Vorfahren und auch den großen Zeitgenossen möglich machen. Die Bildwerke sind Personen von `kultureller Lebendigkeit im Medium der visuellen Materialität.

Damit wird die Frage nach dem ontologischen Status der Bildwerke aufgeworfen. Bildwerke repräsentieren die realen Personen in ihrer konkreten Körperlichkeit gegenüber den lebenden Mitgliedern der Gesellschaft, die den Bildwerken in sozialen Situationen mit ihren eigenen Körpern begegnen, sie wahrnehmen und mit ihnen interagieren. Es ist ein ssozialer U Umgang, der mit aktivem kulturellem Sehen die physische Vergegenwärtigung der dargestellten Götter, Heroen, Verstorbenen und Lebenden bewirkt, mit einem breiten Spektrum der Wirkungen: von Verehrung, Bewunderung und erotischer Anziehung bis zu Erschauern und Schrecken. Zugleich aber bleibt es immer bewusst, dass es Bild-Werke sind, hergestellt von Menschen, aus Materialien, die nicht wie menschliche Wesen belebt sind. Diese ontologische Ambivalenz der Bildwerke, zwischen Lebendigkeit und Materialität, stellt ein Paradox dar, das in das Zentrum der griechischen Bildpraxis führt. Denn die Bildwerke erhalten und entfalten ihre $>$ Realität nicht in der beliebigen Vielfalt der kontingenten Lebenswirklichkeit, sondern in der kulturellen Interaktion zwischen den Mitgliedern der konzeptuellen Gemeinschaft der Lebenden, der Götter und Heroen und der verstorbenen Vorfahren. Sie sind kulturelle Medien, die diesen Umgang in den konstitutiven Situationen des sozialen Lebens möglich machen: den Kult für die Götter und Heroen in den Heiligtümern, die Trauer um die Toten an den Gräbern, die Verehrung und Nachahmung der ruhmvollen Gestalten aus der Vergangenheit und Gegenwart in den öffentlichen Räumen der Städte. In den Bildwerken werden diese Protagonisten der konzeptuellen Gemeinschaft über ihre natürliche Existenz hinaus zu kultureller Bedeutung, das heißt zu Partnern der sozialen und kulturellen Praxis erhoben. ${ }^{40}$

In diesem Sinn kann man das Verhältnis zwischen sozialer Lebenswelt und Bilderwelt reziprok sehen. Einerseits haben die Menschen ihre

40 Zum Verhältnis von `Lebendigkeit` und Materialität der (früh-)griechischen Bildwerke s. die brillanten Überlegungen über »embodiment" bei Neer 2010, 46-57. 
Lebenswelt mit Bildwerken ausgestattet, um die Bedeutung ihrer kulturellen Räume und Praktiken sichtbar zu machen. Andererseits konnten dann die kulturellen Räume mit den Bildwerken eine solche Autonomie erhalten, dass die Menschen gewissermaßen als Besucher und Benutzer der vorgegebenen Bedeutungs-Räume betrachtet werden können. Der Mensch ist immer zugleich Schöpfer und Geschöpf seiner kulturellen Leitbilder. ${ }^{41}$

Aus dieser Perspektive gewinnen nicht zuletzt die formalen Qualitäten der Bildwerke eine neue Aktualität. Die vor allem in Deutschland immer noch verbreitete Befürchtung, Interpretationen von Bildwerken in historischen Kontexten und Funktionen führten an den reigentlich künstlerischen Formen vorbei, ist ganz unbegründet. Im Gegenteil, sie sind unabdingbar für ein Verständnis der Formen, das mehr sein will als einfühlende Beschreibung. Denn wenn Gesellschaften sich Bildwerke als Gestalten der eigenen Lebenswelt, als >Partner` zum Umgang in der eigenen sozialen Praxis schaffen, dann werden die Menschenbilder der 'Kunst` in ihren formalen Qualitäten zu Zeugnissen über diese Gesellschaften, die aus keinem anderen Medium zu gewinnen sind. Fruchtbare Ansätze in diese Richtung sind in der Forschung aufgezeigt worden, sie können hier nicht weiter verfolgt werden. ${ }^{42}$

\section{ABBILDUNGSNACHWEISE}

Abb. 1 Zeichnung T. Hölscher.

Abb. 2 nach Ph. Bruneau - J. Ducat, Guide de Délos 4(Paris 2005) depliant I. Abb. 3 nach R. Hampe - E. Simon, Tausend Jahre frühgriechische Kunst (München 1980) Abb. 450.

Abb. 4 Foto CoDArchLab (Arbeitsstelle für Digitale Archäologie) Universität zu Köln: <http://arachne.uni-koeln.de/item/marbilder/492169>.

Abb. 5 Foto CoDArchLab (Arbeitsstelle für Digitale Archäologie) Universität zu Köln: <http://arachne.uni-koeln.de/item/marbilder/492160>.

Abb. 6 nach G. Kokkorou-Alewras, Die archaische naxische Bildhauerei, AntPl 24 (München 1995) Taf. 11 b.

Abb. 7 nach G. Gruben, Naxos und Delos, JdI 112, 1997, 291 Abb. 13.

Abb. 8 nach G. Kokkorou-Alewras, Die archaische naxische Bildhauerei, AntPl 24 (München 1995) Taf. 9.

$41 \mathrm{Zu}$ dieser Reziprozität s. Muth 1998.

42 Neuere Beispiele: Hölscher 2010; Neer 2010; Borbein 2014. 
Abb. 9 nach M. Maass, Das antike Delphi (Darmstadt 1993) Plan 2.

Abb. 10 nach P. Amandry, La Colonne des Naxiens et le Portique des Athéniens. Fouilles de Delphes 2 (Paris 1953) Taf. 14.

Abb. 11 nach G. Kokkorou-Alewras, Die archaische naxische Bildhauerei, AntPl 24 (München 1995) Taf. 54 a

Abb. 12 nach P. C. Bol (Hrsg.), Die Geschichte der antiken Bildhauerkunst 1 (Mainz 2002) Abb. 212 b

Abb. 13 nach K. Karakasi, Archaische Koren (München 2001) Taf. 29.

Abb. 14 nach J. Franssen, Votiv und Repräsentation. Statuarische Weihungen archaischer Zeit aus Samos und Attika (Heidelberg 2011) Taf. 1, 1.

Abb. 15 nach H. Kyrieleis, Der große Kuros von Samos. Samos 10 (Bonn 1996) Taf. 16.

Abb. 16 nach G. M. A. Richter, The Archaic Gravestones of Attica (London 1961) Abb. 99.

Abb. 17 A. Borbein, Das alte Griechenland. Geschichte und Kultur der Hellenen (München 1995) 257.

Abb. $18 \mathrm{H}$. A. Shapiro, Art and Cult under the Tyrants in Athens (Mainz 1989) 9 Abb. 3.

Abb. 19 Deutsches Archäologisches Institut Rom.

Abb. 20 nach P. C. Bol (Hrsg.), Die Geschichte der antiken Bildhauerkunst 1 (Mainz 2002) Abb. 157 a

Abb. 21 nach R. Neer, Art and Archaeology of the Greek World (London 2012) Abb. 4, 32.

Abb. 22 Phototek, Archäologisches Institut der Friedrich-Wilhelms-Universität Bonn.

\section{LITERATURVERZEICHNIS}

Die Abkürzungen der Periodika und Zeitschriften richten sich nach den Richtlinien des Deutschen Archäologischen Instituts (www.dainst.org/ medien/de/richtlinien_abkuerzungen.html); die der antiken Autoren nach dem Verzeichnis im Neuen Pauly (Bd. 1 und 3).

\section{Borbein 2014}

A. H. Borbein, Das Medium der künstlerischen Form. Medium - künstlerische Form - Geschichte. Drei Begriffe und zugleich drei Problemfelder, in: O. Dally u. a. (Hrsg.), Medien der Geschichte. Antikes Griechenland und Rom (Berlin 2014) 244-253.

\section{Borell - Rittig 1998}

B. Borell - D. Rittig, Orientalische und griechische Bronzereliefs aus Olympia, OF 26 (Berlin 1998). 


\section{Brisart 2011}

Th. Brisart, Un art citoyen. Recherches sur l'orientalisation des artisanats en Grece proto-archaique (Brüssel 2011).

\section{Bruneau - Ducat 2005}

Ph. Bruneau - J. Ducat, Guide de Délos (Paris 2005).

\section{Brunnsåker 1953}

St. Brunnsåker, The Tyrant-Slayers of Kritios and Nesiotes (Lund 1953).

\section{Bumke 2004}

H. Bumke, Statuarische Gruppen in der frühen griechischen Kunst (Berlin 2004).

\section{D'Acunto 1995}

M. D’Acunto, I cavalieri di Priniàs e il tempio A, AnnOrNapFil 2, 15-56.

\section{D'Acunto 2008}

M. D'Acunto, La fonction de la plus ancienne sculpture naxienne à Délos et la comparaison avec les productions crétoises dédaliques. Sur les débuts de la sculpture monumentale en Grèce, in: G. Kuragios - F. Prost (Hrsg.), La sculpture des Cyclades à l'époque archaique. Histoire des ateliers, rayonnement des styles. Actes du colloque international organisé par l'Éphorie des Antiquités préhistoriques et classiques des Cyclades et l'École française d'Athènes, 7-9 septembre 1998, BCH Suppl. 48 (Athen 2008) 133-182.

\section{Daehner 2005}

J. Daehner, Grenzen der Nacktheit. Studien zum nackten männlichen Körper in der griechischen Plastik des 5. und 4. Jahrhunderts v. Chr., JdI 120, 2005, 155-299.

\section{D'Onofrio 1982}

A. M. D’Onofrio, Kouroi e korai funerari attici, AnnOrNapFil 4, 1982, 135170 .

\section{Donohue 1988}

A. A. Donohue, Xoana and the Origins of Greek Sculpture (Atlanta 1988).

\section{Franssen 2011}

J. Franssen, Votiv und Repräsentation. Statuarische Weihungen archaischer Zeit aus Samos und Attika (Heidelberg 2011).

\section{Fuchs - Floren 1987}

W. Fuchs - J. Floren, Die griechische Plastik 1. Die geometrische und archaische Plastik (München 1987).

\section{Giuliani 2005}

L. Giuliani, Der Koloss der Naxier, in: L. Giuliani (Hrsg.), Meisterwerke der antiken Kunst (München 2005) 12-27. 


\section{Gruben 1997}

G. Gruben, Naxos und Delos. Studien zur archaischen Architektur der Kykladen, JdI 117, 1997, 261-416.

\section{Gruben 2001}

G. Gruben, Griechische Tempel und Heiligtümer 5(München 2001).

\section{Gunter 2009}

A. C. Gunter, Greek Art and the Orient (Cambridge 2009).

\section{Herrmann 1975}

V.-V. Herrmann, Zum Problem der Entstehung der griechischen Großplastik, in: Wandlungen. Studien zur antiken und neueren Kunst. Ernst Homann-Wedeking gewidmet (Waldsassen 1975) 35-48.

\section{Himmelmann 1964}

N. Himmelmann, Bemerkungen zur geometrischen Figur (Berlin 1964).

\section{Himmelmann 1990}

N. Himmelmann, Ideale Nacktheit in der griechischen Kunst (Berlin 1990).

\section{Hölscher 1972}

F. Hölscher, Die Bedeutung archaischer Tierkampfbilder (Würzburg 1972).

\section{Hölscher 1998}

T. Hölscher, Öffentliche Räume in frühen griechischen Städten ${ }^{2}$ (Heidelberg 1998).

\section{Hölscher 1999}

T. Hölscher, Immagini mitologiche e valori sociali nella Grecia arcaica, in:

F. De Angelis - S. Muth (Hrsg.), Im Spiegel des Mythos. Bilderwelt und Lebenswelt (Wiesbaden 1999) 11-30.

\section{Hölscher 2002}

T. Hölscher, Rituelle Räume und politische Denkmäler im Heiligtum von Olympia, in: H. Kyrieleis (Hrsg.), Olympia 1875-2000. 125 Jahre deutsche Ausgrabungen (Mainz 2002) 347-358.

\section{Hölscher 2003}

T. Hölscher, Körper, Handlung und Raum als Sinnfiguren in der griechischen Kunst und Kultur, in: K.-J. Hölkeskamp u. a. (Hrsg.), Sinn (in) der Antike. Orientierungssysteme, Leitbilder und Wertkonzepte im Altertum (Mainz 2003) 163-192.

\section{Hölscher 2007}

T. Hölscher, Die Griechische Kunst (München 2007).

\section{Hölscher 2010}

T. Hölscher, Menschenbilder in der altgriechischen Kunst, in: M. Hilgert M. Wink (Hrsg.), Menschenbilder. Darstellungen des Humanen in der Wissenschaft, Heidelberger Jahrbücher 54 (Heidelberg 2010) 27-47. 


\section{Hölscher 2012}

T. Hölscher, Bilderwelt, Lebensordnung und die Rolle des Betrachters im antiken Griechenland, in: O. Dally u. a. (Hrsg.), Bild - Raum - Handlung. Perspektiven der Archäologie (Berlin 2012) 19-44.

\section{Homann-Wedeking 1950}

E. Homann-Wedeking, Die Anfänge der griechischen Großplastik (Berlin 1950).

\section{Hurwit 2007}

J. M. Hurwit, The Problem with Dexileos. Heroic and Other Nudities in Greek Art, AJA 111, 2007, 35-60.

\section{Jacquemin 1999}

A. Jacquemin, Offrandes monumentales à Delphes (Paris 1999).

\section{Kissas 2000}

K. Kissas, Die attischen Statuen- und Stelenbasen archaischer Zeit (Bonn 2000).

\section{Kokkorou-Alewras 1995}

G. Kokkorou-Alewras, Die archaische naxische Bildhauerei, AntPl 24 (München 1995) 37-133.

\section{Krahmer 1931}

G. Krahmer, Figur und Raum in der ägyptischen und griechisch-archaischen Kunst (Halle 1931).

\section{Kyrieleis 1996}

H. Kyrieleis, Der große Kouros von Samos, Samos 10 (Bonn 1996).

\section{Martini 1990}

W. Martini, Die archaische Plastik der Griechen (Darmstadt 1990).

\section{Meier 1985}

Chr. Meier, Politik und Anmut (Berlin 1985).

\section{Meyer - Brüggemann 2007}

M. Meyer - N. Brüggemann, Kore und Kouros. Weihegaben für die Götter (Wien 2007).

\section{Morris 1996}

I. Morris, The Strong Principle of Equality and the Archaic Origins of Greek Democracy, in: J. Ober - C. Hedrick (Hrsg.), Demokratia. A Conversation on Democracies, Ancient and Modern (Princeton 1996) 19-48.

\section{Muth 1998}

S. Muth, Erleben von Raum - Leben im Raum. Zur Funktion mythologischer Mosaikbilder in der römisch-kaiserzeitlichen Wohnarchitektur (Heidelberg 1998). 


\section{Neer 2001}

R. Neer, Framing the Gift. The Politics of the Siphnian treasury at Delphi, Classical Antiquity 20, 2001, 273-336.

\section{Neer 2010}

R. Neer, The Emergence of the Classical Style in Greek Sculpture (Chicago 2010).

\section{Neer 2012}

R. Neer, Art and Archaeology of the Greek World (London 2012).

\section{Picard 1991}

O. Picard, Guide de Delphes. Le Musée (Athen 1991).

\section{Richter 1961}

G. M. A. Richter, The Archaic Gravestones of Attica (London 1961).

\section{Ridgway 1977}

B. S. Ridgway, The Archaic Style in Greek Sculpture (Princeton 1977).

\section{Rolley 1994}

C. Rolley, La sculpture grecque 1 (Paris 1994).

\section{Romano 1980}

I. B. Romano, Early Greek Cult Images (Pennsylvania 1980).

\section{Scheibler 1979}

I. Scheibler, Griechische Künstlervotive der archaischen Zeit, MüJb 30, 1979, 7-30.

\section{Schneider 1975}

L. Schneider, Zur sozialen Bedeutung der archaischen Korenstatuen (Hamburg 1975).

\section{Stewart 1990}

A. Stewart, Greek Sculpture. An Exploration (New Haven 1990).

\section{Vernant 1985}

J.-P. Vernant, Mythe et pensée chez les Grecs (Paris 1985).

\section{Vernant 1990}

J.-P. Vernant, Figures, idoles, masques (Paris 1990).

\section{Vorster 2002}

Chr. Vorster, Früharchaische Plastik, in: P. C. Bol (Hrsg.), Die Geschichte der antiken Bildhauerkunst 1. Frühgriechische Plastik (Mainz 2002) 97-132.

\section{Winkler-Horacek 2000}

L. Winkler-Horacek, Mischwesen und Tierfries in der archaischen Vasenmalerei von Korinth, in: T. Hölscher (Hrsg.), Gegenwelten zu den Kulturen Griechenlands und Roms in der Antike (München 2000) 217-244. 